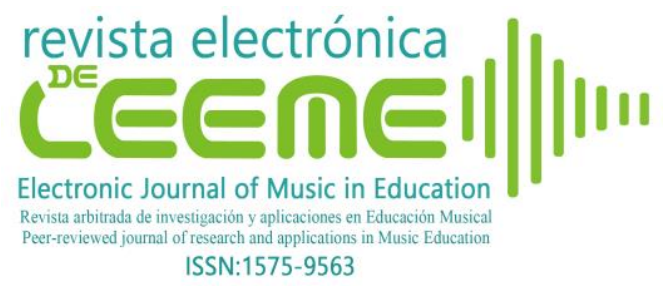

\title{
Cuando el barrio educa: aprendizaje situado y creación artística colaborativa como herramienta en la formación musical del futuro docente
}

\section{When the neighborhood educates: situated learning and collaborative artistic creation as a tool in the musical formation of the future teacher}

\author{
Noemy Berbel Gómez \\ noemy.berbel@uib.es \\ Departamento de Pedagogía y Didácticas Específicas \\ Universidad de las Islas Baleares \\ Palma de Mallorca, España \\ ORCID: http://orcid.org/0000-0001-8125-9679

$\begin{array}{r}\text { Adolf Murillo Ribes } \\ \text { adolf.murillo@ uv.es }\end{array}$
Instituto de Creatividad e Innovaciones Educativas
Universidad de Valencia
Valencia, España

Ma Elena Riaño Galán

elena.riano@unican.es

Departamento de Educación

Universidad de Cantabria

Santander, España

ORCID: http://orcid.org/0000-0002-8274-9917

doi:10.7203/LEEME.46.17764

Recibido: 28-06-2020 Aceptado: 29-07-2020. Contacto y correspondencia: Adolf Murillo. Departamento de Didáctica de la Expresión Musical, Plástica y Corporal, Universidad de Valencia, Avda. dels Tarongers, 4, 46022. Valencia. España.

\section{Resumen}

Este estudio describe y analiza una acción artístico-pedagógica en el ámbito de la formación inicial de docentes y desarrollada en un barrio desfavorecido de Palma de Mallorca, España. Participaron 65 estudiantes del Grado de Maestro de Educación Primaria en la asignatura de Música y 3 músicos expertos, responsables de diseñar e implementar la acción. A través de una metodología cualitativa de tipo descriptivo-interpretativo, los resultados muestran: 1) algunas características que determinaron el diseño de la acción como son el aprendizaje situado, la colaboración, la creatividad y el compromiso con la realidad social del barrio; y 2) aspectos emergentes a partir de las valoraciones de los participantes relacionados con las dimensiones metodológica, creativa y social. Las conclusiones sugieren que este tipo de acciones son una oportunidad en la formación, tanto disciplinar como pedagógica, de los futuros docentes, sean estos generalistas o especialistas de Música. Es necesario continuar indagando sobre estrategias docentes que les ayude a poner en práctica experiencias artísticas contextualizadas desde un enfoque creativo y colaborativo.

Palabras clave: Formación inicial; educación musical; creatividad; aprendizaje situado.

\section{Abstract}

This study describes and analyzes an artistic-pedagogical action in the field of initial teacher training and developed in a disadvantaged neighborhood of Palma de Mallorca, Spain. 65 students of the Teaching Degree (Primary) in the subject of music and three expert musicians, responsible for designing and implementing the action, participated. Through a qualitative methodology of descriptive interpretation, the results show: 1) some characteristics that determined the design of the action such as situated learning, collaboration, creativity, and commitment with the social reality of the neighborhood; and 2) emerging aspects from the participants' evaluations related to the methodological, creative, and social dimensions. The conclusions suggest that this type of action is an opportunity in the training, both disciplinary and pedagogical, of future teachers, whether they are generalists or specialists in music. It is necessary to continue investigating teaching strategies that help them to put into practice contextualized artistic experiences from a creative and collaborative approach.

Key words: Initial training; music education; creativity; situated learning.

@ Noemy Berbel Gómez, Adolf Murillo Ribes y Mª Elena Riaño Galán. The content of this article is the sole responsibility of the authors. The Revista Electrónica de LEEME and Universitat de València are not liable for any legal actions that may arise involving the article's content. Revista Electrónica de LEEME - Lista Electrónica Europea de Música en la Educación-. http://ojs.uv.es/index/php/LEEME/index ISSN: 1575-9563. Editores: Universidad de Valencia y Jesús Tejada. Visibilidad de esta revista: SCOPUS, Emerging Sources Citation Index (Clarivate), EBSCO, CINDOC (CSIC), Citefactor, COPAC, Dialnet, DICE (CSIC), DOAJ, e-revistas (CSIC), EBSCO Premier, ERIH+, Gale Cengage Learning, IN-RECS, IRESIE, LATINDEX, MIAR, OCLC Worldcat, RESH, REDIB, RILM Core Journals, SUDOC, ULRICHS. Esta revista es de acceso libre mediante licencia Creative Commons 4.0 CC by. Política de archivado: etiqueta verde SHERPA-ROMEO. 


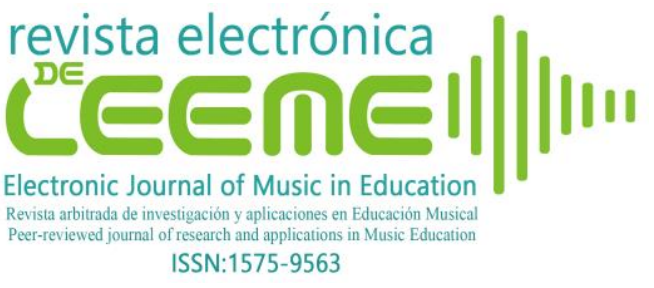

Noemy Berbel Gómez, Adolf Murillo Ribes y Mª Elena Riaño Galán Cuando el barrio educa: aprendizaje situado y creación artística colaborativa como herramienta en la formación musical (Revista Electrónica de LEEME) Número 46, pp. 68-91 https://ojs.uv.es/index.php/LEEME/index ISSN:1575-9563

\section{Introducción}

Desde la llegada del Plan Bolonia, la formación inicial del profesorado en la universidad supuso un cambio de un modelo basado en la enseñanza a otro basado en el aprendizaje que situó al alumnado en el centro de su proceso, favoreciendo su implicación, actividad y protagonismo (Mayorga y Madrid, 2010). En este contexto, es necesario dotar al futuro profesorado, tanto generalista como especialista de Educación Musical de experiencias significativas en las que participen activamente y cuyas voces sean tenidas en cuenta (O'Neill, 2015).

Para que esto sea así, las prácticas en las aulas han de basarse en modelos educativos integradores en los que lo disciplinar y pedagógico se interrelacionen, planteadas también en contextos situados reales donde el alumnado construya su conocimiento participando y colaborando de experiencias musicales creativas (Kenny, Finnern y Mitchell, 2015). Las experiencias de inmersión "en la vida real" que tienen en cuenta la diversidad, junto con una profunda reflexión sobre la práctica, provocan cambios en los enfoques pedagógicos y en las relaciones entre docentes y alumnado (Abrahams, Rowland y Kohler, 2012).

Por todo ello, este estudio se centra en visibilizar y profundizar posibles estrategias docentes que ayuden a los futuros docentes generalistas y especialistas de Música a poner en práctica experiencias artísticas contextualizadas desde un enfoque creativo y colaborativo. Se pretende conocer las percepciones de un grupo de alumnado universitario en el ámbito de la Educación Musical, futuros docentes generalistas de Educación Primaria, participantes en una acción pedagógica basada en la creación sonora colaborativa y contextualizada en una situación real.

\section{Marco teórico}

\subsection{Formación inicial de docentes desde la educación musical en contexto.}

La educación musical ha estado presente en los currículos de acuerdo con el sistema educativo español desde hace décadas y su enseñanza ha sido labor del profesorado especialista. Sin embargo, la compleja sociedad, caracterizada por una multiplicidad de realidades que implican resolver problemas día a día, demanda la formación de docentes generalistas que sean competentes en lo disciplinario y lo pedagógico al mismo tiempo; docentes capaces de ampliar su mirada y abordar perspectivas pedagógicas nuevas que incluyan enfoques transdisciplinares.

@ Noemy Berbel Gómez, Adolf Murillo Ribes y Mª Elena Riaño Galán. The content of this article is the sole responsibility of the authors. The Revista Electrónica de LEEME and Universitat de València are not liable for any legal actions that may arise involving the article's content. Revista Electrónica de LEEME - Lista Electrónica Europea de Música en la Educación-. http://ojs.uv.es/index/php/LEEME/index ISSN: 1575-9563. Editores: Universidad de Valencia y Jesús Tejada. Visibilidad de esta revista: SCOPUS, Emerging Sources Citation Index (Clarivate), EBSCO, CINDOC (CSIC), Citefactor, COPAC, Dialnet, DICE (CSIC), DOAJ, e-revistas (CSIC), EBSCO Premier, ERIH+, Gale Cengage Learning, IN-RECS, IRESIE, LATINDEX, MIAR, OCLC Worldcat, RESH, REDIB, RILM Core Journals, SUDOC, ULRICHS. Esta revista es de acceso libre mediante licencia Creative Commons 4.0 CC by. Política de archivado: etiqueta verde SHERPA-ROMEO. 


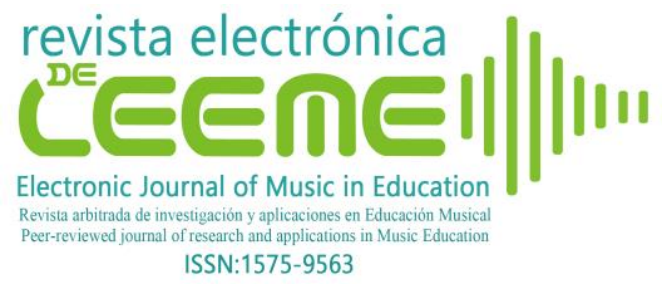

Noemy Berbel Gómez, Adolf Murillo Ribes y Mª Elena Riaño Galán Cuando el barrio educa: aprendizaje situado y creación artística colaborativa como herramienta en la formación musical (Revista Electrónica de LEEME) Número 46, pp. 68-91 https://ojs.uv.es/index.php/LEEME/index ISSN:1575-9563

La Música, al igual que cualquier otra disciplina artística puede ser desarrollada por docentes generalistas desde enfoques más abiertos, participativos o multidisciplinares (Young, 2018). Los objetivos de la educación musical y artística pueden diferir considerablemente dependiendo de que la educación musical esté impartida por especialistas o por generalistas (Aróstegui y Cisneros-Cohernour, 2010). Sin embargo, la calidad cuestionable de la enseñanza de la música por parte del profesorado de Educación Primaria generalista se ha identificado tanto a nivel nacional como internacional (de Vries, 2013). Esto ocurre cuando la Música se entiende como un área auxiliar o complemento de otras áreas del conocimiento (Wiggins, 2008), cuando hay falta de confianza por parte del profesorado generalista (Biasutti, Hennessy y de Vugt-Jansen 2015; Mota, 2015) o cuando se ofrecen limitadas experiencias musicales en su formación inicial (Hennessy 2017; Welch y Henley 2014). Esta misma calidad se cuestiona igualmente cuando el profesorado es especialista, pero existe el riesgo de una enseñanza basada en la mera reproducción cultural, debida al hecho que se ven a sí mismos como músicos antes que como docentes (Aróstegui y Cisneros-Cohernour, 2010).

En este estudio, se pone el foco en la educación musical que el profesorado generalista recibe en el contexto de su formación inicial. Debe tenerse en cuenta que la formación que reciba tendrá repercusión en su futura práctica docente. Puede ocurrir que un docente generalista pueda centrarse más en la parte académica de los contenidos musicales que en la parte estética, o percibir que el tiempo dedicado a la Música supone mermar las disciplinas «básicas» (Bresler, 1993). La falta de conocimiento musical se cita a menudo como un problema para el profesorado generalista de Primaria. Este colectivo además del conocimiento musical necesita disponer de la confianza y las habilidades necesarias para enseñar esta disciplina (de Vries 2014). No obstante, existen estudios que demuestran que los adultos tienen capacidad para desarrollarse musicalmente a través de oportunidades que les permitan participar en la creación musical (Henley 2015), en lugar de concebir la habilidad musical como una entidad fija (Biasutti 2010). La autoeficacia docente supone, así, un elemento clave en la mejora de la confianza del docente generalista en su tarea de enseñar música. Esto, a su vez, es un factor motivador para que el profesorado continúe su labor educativa (de Vries, 2013).

\subsection{Enfoques creativos y metodologías}

Los enfoques pedagógicos creativos y reflexivos son fundamentales para reforzar las identidades, valores y enfoques de la educación musical en las escuelas, desafiando al alumnado a pensar (Burnard, 2012). La educación, desde una perspectiva creativa, implica una participación y un pensamiento positivo abierto a las posibilidades (Craft 2005); también implica un alto uso de medios de comunicación y múltiples alfabetizaciones, permitiendo así que el paisaje de la diversidad forme parte del espacio del aula (Craft, 2012). Un espacio

@ Noemy Berbel Gómez, Adolf Murillo Ribes y Mª Elena Riaño Galán. The content of this article is the sole responsibility of the authors. The Revista Electrónica de LEEME and Universitat de València are not liable for any legal actions that may arise involving the article's content. Revista Electrónica de LEEME - Lista Electrónica Europea de Música en la Educación-. http://ojs.uv.es/index/php/LEEME/index ISSN: 1575-9563. Editores: Universidad de Valencia y Jesús Tejada. Visibilidad de esta revista: SCOPUS, Emerging Sources Citation Index (Clarivate), EBSCO, CINDOC (CSIC), Citefactor, COPAC, Dialnet, DICE (CSIC), DOAJ, e-revistas (CSIC), EBSCO Premier, ERIH+, Gale Cengage Learning, IN-RECS, IRESIE, LATINDEX, MIAR, OCLC Worldcat, RESH, REDIB, RILM Core Journals, SUDOC, ULRICHS. Esta revista es de acceso libre mediante licencia Creative Commons 4.0 CC by. Política de archivado: etiqueta verde SHERPA-ROMEO. 


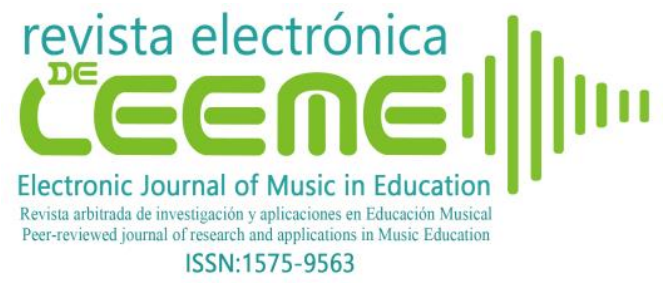

Noemy Berbel Gómez, Adolf Murillo Ribes y Mª Elena Riaño Galán Cuando el barrio educa: aprendizaje situado y creación artística colaborativa como herramienta en la formación musical (Revista Electrónica de LEEME) Número 46, pp. 68-91 https://ojs.uv.es/index.php/LEEME/index ISSN:1575-9563

dialógico definido por Wegerif (2013) como multidimensional, dinámico y continuo de significado que abre la puerta a nuevas percepciones sobre el proceso de aprendizaje y de la enseñanza creativa en la educación musical.

Según un reciente estudio realizado en Irlanda, las experiencias musicales creativas y de colaboración facilitaron nuevos entendimientos de las pedagogías y prácticas de educación musical, desafiando en las escuelas las ideas preconcebidas sobre la enseñanza de la música (Kenny, 2015). En otros estudios, se demostró que las personas que dedicaban más tiempo a la exploración de materiales antes de finalizar los productos lograban resultados más creativos que aquellas personas que dedicaban menos tiempo a la exploración (Brinkman, 2010). La exploración puede explicarse mediante el uso de términos como búsqueda, variación, asunción de riesgos, experimentación, juego, flexibilidad, descubrimiento e innovación. Este proceso puede crear en el alumnado una disposición de "necesidad de saber" que generará curiosidad por un aprendizaje más técnico (Hickey, 2012). Asimismo, la exploración implica buscar cambios, tomar riesgos y empujar a los experimentos (Marsh y Marsh, 2008; Marsch y Young, 2006). Proporcionar espacios y tiempo es esencial dentro de la educación musical para que se produzca esa creación musical en grupo a fin de crear prácticas musicales creativas compartidas; de esta manera, el alumnado podrá adoptar tales prácticas pedagógicas creativas en sus propias aulas futuras. Encontrar formas de facilitar y mejorar las oportunidades de reflexión profunda sobre las experiencias prácticas debería estar a la vanguardia de los cursos de educación musical para asegurar que se establezcan esas conexiones (Kenny, 2015).

El Aprendizaje Basado en la Creación (en adelante, $\mathrm{ABC}$ ) supone una vivencia en la cual el discente se proyecta en la acción creadora. Caiero (2018) define el ABC como:

\begin{abstract}
[...] una vivencia educativa, cognitiva, sensitiva y emotiva cuyo propósito puede ser construir un objeto, generar un producto o dar forma física a una idea o a un sentimiento por medio de lenguajes, materiales, herramientas y recursos diversos pasando por acciones y fases que le dan sentido, donde el crear adquiere protagonismo y cuyo desencadenante de todo ese aprendizaje (en el caso del arte) es el deseo. Este tipo de acción educativa supone la organización de la vida misma del alumno en una forma visible que nos transmite a la comunidad algo que es al mismo tiempo cultural, personal y contemporáneo (p.160).
\end{abstract}

El ABC comparte aspectos con el Aprendizaje Basado en Proyectos (ABP, en adelante), pues ambos buscan un aprender activo y significativo (Vergara, 2015). En el caso del ABC, este permite integrar las emociones, sentimientos e ideas a través de los elementos artísticos y una proyección no solo hacia un destino o solución preconcebida, como ocurre en la mayoría de los proyectos fundamentados en tareas programadas, problemas ya resueltos y gramáticas ya conclusas (Caeiro, 2018). Así pues, se debe alentar al profesorado a correr riesgos, experimentando con diversas ideas, repertorios y enfoques sin temor a fracasar, y a que se comprometan ellos mismos en procesos creativos que "tienen muchas situaciones inesperadas,

@ Noemy Berbel Gómez, Adolf Murillo Ribes y Mª Elena Riaño Galán. The content of this article is the sole responsibility of the authors. The Revista Electrónica de LEEME and Universitat de València are not liable for any legal actions that may arise involving the article's content. Revista Electrónica de LEEME - Lista Electrónica Europea de Música en la Educación-. http://ojs.uv.es/index/php/LEEME/index ISSN: 1575-9563. Editores: Universidad de Valencia y Jesús Tejada. Visibilidad de esta revista: SCOPUS, Emerging Sources Citation Index (Clarivate), EBSCO, CINDOC (CSIC), Citefactor, COPAC, Dialnet, DICE (CSIC), DOAJ, e-revistas (CSIC), EBSCO Premier, ERIH+, Gale Cengage Learning, IN-RECS, IRESIE, LATINDEX, MIAR, OCLC Worldcat, RESH, REDIB, RILM Core Journals, SUDOC, ULRICHS. Esta revista es de acceso libre mediante licencia Creative Commons 4.0 CC by. Política de archivado: etiqueta verde SHERPA-ROMEO. 


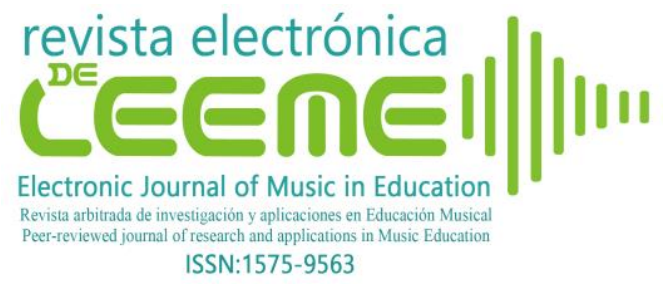

Noemy Berbel Gómez, Adolf Murillo Ribes y Mª Elena Riaño Galán Cuando el barrio educa: aprendizaje situado y creación artística colaborativa como herramienta en la formación musical (Revista Electrónica de LEEME) Número 46, pp. 68-91 https://ojs.uv.es/index.php/LEEME/index ISSN:1575-9563

inciertas e impredecibles que dan lugar a la incertidumbre, la motivación, la sorpresa y el deseo de recorrer atajos y caminos inexplorados" (Elisondo, Donolo y Rinaudo, 2013, p.13).

\subsection{Aprendizaje situado y transformación}

Una de las claves en la formación inicial del profesorado es incluir experiencias de "aprendizaje situado" para desarrollar comunidades discursivas de aprendizaje. En este modelo situado el alumnado está más inclinado a aprender participando activamente en la experiencia de aprendizaje. Se trata esencialmente de crear un significado a partir de las experiencias de la vida diaria en un contexto real (Lave y Wenger, 1991). De esta manera, ampliarán sus oportunidades para "descubrir" el éxito de la enseñanza a diversas poblaciones de alumnado de contextos diversos (Robinson, 2016). De acuerdo con algunas investigaciones relacionadas con la participación en experiencias reales, las personas pueden mostrar altos grados de motivación, a la vez que experimentan un sentido de compromiso y conexión con la comunidad escolar (Reynolds, Preston y Haynes, 2005). Son oportunidades que contribuyen a desarrollar la competencia cultural y la socialización de los profesores (McKoy, 2013).

El profesorado generalista, en su formación inicial, suele mostrar muy poco conocimiento de algunos problemas presentes en la sociedad actual de carácter intercultural y tampoco tienen experiencias directas, o si las tienen son muy limitadas (Chou, 2007). En un estudio realizado por Amatea, Cholewa y Mixon (2012), se mostró que el maestro promedio (raza blanca) generalmente tiene experiencias de vida muy diferentes y una comprensión inadecuada de las perspectivas y circunstancias de la vida del alumnado de bajos ingresos y/o de minorías étnicas. Por tanto, se requiere dotar al futuro profesorado de experiencias de aprendizaje basadas en contextos reales (Salvador-Tomàs, Jaume-Androver y Berbel-Gómez, 2019).

Para proporcionar a las escuelas experiencias de enseñanza y aprendizaje que sean ricas y auténticas es necesario que los formadores de docentes de música se comprometan a crear asociaciones sólidas con las escuelas y las organizaciones comunitarias (Doberneck, Glass y Schweitzer, 2010). Estos vínculos requieren una visión estratégica, una preparación reflexiva y el coraje de ir más allá de la concepción tradicional de los roles en la escuela, potenciando el trabajo de campo frente a la mera observación (Forrester, 2019). Por esto, es necesario dotar tanto al futuro docente generalista como al especialista de experiencias artísticas significativas que vayan más allá de las destrezas instrumentales.

El objetivo de este estudio ha sido conocer las percepciones de futuros docentes generalistas de Educación Primaria en el ámbito de la Educación Musical sobre la relevancia de

@ Noemy Berbel Gómez, Adolf Murillo Ribes y Mª Elena Riaño Galán. The content of this article is the sole responsibility of the authors. The Revista Electrónica de LEEME and Universitat de València are not liable for any legal actions that may arise involving the article's content. Revista Electrónica de LEEME - Lista Electrónica Europea de Música en la Educación-. http://ojs.uv.es/index/php/LEEME/index ISSN: 1575-9563. Editores: Universidad de Valencia y Jesús Tejada. Visibilidad de esta revista: SCOPUS, Emerging Sources Citation Index (Clarivate), EBSCO, CINDOC (CSIC), Citefactor, COPAC, Dialnet, DICE (CSIC), DOAJ, e-revistas (CSIC), EBSCO Premier, ERIH+, Gale Cengage Learning, IN-RECS, IRESIE, LATINDEX, MIAR, OCLC Worldcat, RESH, REDIB, RILM Core Journals, SUDOC, ULRICHS. Esta revista es de acceso libre mediante licencia Creative Commons 4.0 CC by. Política de archivado: etiqueta verde SHERPA-ROMEO. 


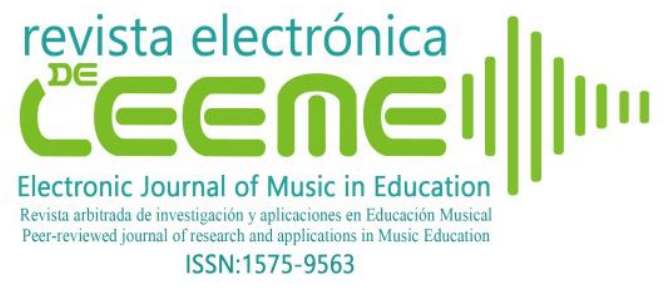

Noemy Berbel Gómez, Adolf Murillo Ribes y Mª Elena Riaño Galán Cuando el barrio educa: aprendizaje situado y creación artística colaborativa como herramienta en la formación musical (Revista Electrónica de LEEME) Número 46, pp. 68-91 https://ojs.uv.es/index.php/LEEME/index

ISSN:1575-9563

una acción pedagógica en la que participaron, que fue basada en la creación sonora colaborativa y contextualizada en una situación real.

\section{Método}

\subsection{Contexto del estudio}

En primer lugar, cabe señalar que la motivación de este estudio se deriva de la participación de los autores en un proyecto de investigación que parte de un supuesto: que las experiencias con el arte, mediante procesos creativos de participación, son capaces de contribuir a la construcción de un entramado social que refuerce el tejido vecinal, educativo y cultural. El programa aquí descrito se desarrolla en el barrio desfavorecido y marginal de Nou Llevant/Soletat Sud de Palma de Mallorca a partir del diseño de la acción titulada: 'Sonorización del Barrio: El Latido Sonoro'.

\subsection{Diseño}

Se siguió una metodología cualitativa (Gibbs, 2012) de tipo descriptivo-interpretativo y de corte etnográfico (Hammersley y Atkinson, 2009). Las técnicas de investigación utilizadas fueron la observación participante sobre el desarrollo de la acción artístico-pedagógica, así como el grupo de discusión con los participantes con respecto a su opinión sobre los aspectos más relevantes en dicha acción (Creswell, 2013). El grupo de discusión fue guiado por una serie de preguntas que sirvieron como detonante ¿qué ha significado para ti participar en esta experiencia? Tras tu participación en la experiencia ¿qué reflexiones podrían ser determinantes en tu futuro profesional? dar la oportunidad al alumnado de expresarse con plena libertad. La sesión fue registrada en video. Se transcribieron las opiniones y luego fueron analizadas y categorizadas (Dey, 2005).

\subsection{Participantes}

De los 68 participantes, 65 eran estudiantes del Grado de Maestro de Educación Primaria de la Universidad de las Islas Baleares que cursaban la asignatura de "Educación Artística: Música. Didáctica en la Escuela Primaria” (el 74,5 \% eran mujeres y 25,5 \% hombres) y los 3 restantes eran músicos/expertos responsables de diseñar e implementar la acción pedagógica (autores del artículo) y también observadores participantes durante la experiencia. Uno de estos expertos era el docente responsable de impartir la asignatura universitaria y los otros dos eran expertos invitados específicamente para la acción. Asimismo, la experiencia se ha

@ Noemy Berbel Gómez, Adolf Murillo Ribes y Mª Elena Riaño Galán. The content of this article is the sole responsibility of the authors. The Revista Electrónica de LEEME and Universitat de València are not liable for any legal actions that may arise involving the article's content. Revista Electrónica de LEEME - Lista Electrónica Europea de Música en la Educación-. http://ojs.uv.es/index/php/LEEME/index ISSN: 1575-9563. Editores: Universidad de Valencia y Jesús Tejada. Visibilidad de esta revista: SCOPUS, Emerging Sources Citation Index (Clarivate), EBSCO, CINDOC (CSIC), Citefactor, COPAC, Dialnet, DICE (CSIC), DOAJ, e-revistas (CSIC), EBSCO Premier, ERIH+, Gale Cengage Learning, IN-RECS, IRESIE, LATINDEX, MIAR, OCLC Worldcat, RESH, REDIB, RILM Core Journals, SUDOC, ULRICHS. Esta revista es de acceso libre mediante licencia Creative Commons 4.0 CC by. Política de archivado: etiqueta verde SHERPA-ROMEO. 


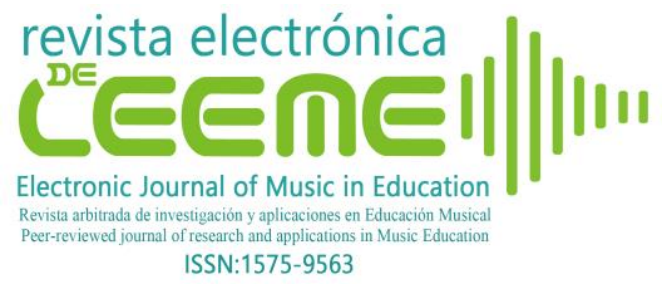

Noemy Berbel Gómez, Adolf Murillo Ribes y Mª Elena Riaño Galán Cuando el barrio educa: aprendizaje situado y creación artística colaborativa como herramienta en la formación musical (Revista Electrónica de LEEME) Número 46, pp. 68-91 https://ojs.uv.es/index.php/LEEME/index ISSN:1575-9563

llevado a cabo con alumnado de Educación Primaria del Colegio Público Pintor Joan Miró de Palma de Mallorca. No obstante, aunque participaron en la acción, no se incluyeron como parte de este estudio, ya que el interés del estudio se centró en la formación inicial del profesorado.

\subsection{Procedimiento}

El trabajo desarrollado en el diseño, implementación y reflexión de la acción artísticopedagógica se llevó a cabo a lo largo de 4 meses dentro del horario lectivo. Durante este tiempo, se realizaron dos talleres y un grupo de discusión. Los talleres se desarrollaron a lo largo de tres sesiones cada uno con una duración de tres horas por sesión. La primera se realizó con el alumnado de la facultad en el Laboratorio de Música y Arte Mus\&Art LAB y fue una sesión preparatoria donde se indicaron unas pautas para la tutorización del trabajo de campo (captura sonidos del barrio) con el alumnado de Educación Primaria. El profesorado en formación también se familiarizó con el uso de las grabadoras digitales y la tecnología de mapeo ${ }^{1}$. La segunda sesión se llevó a cabo en el centro escolar y en el barrio, con discentes de cuarto curso de Educación Primaria junto el alumnado universitario. En esta sesión, se llevó a cabo todo el trabajo de campo de grabación e intervención en el espacio del barrio; la tercera sesión se realizó de nuevo en la universidad, donde trabajaron conjuntamente el alumnado universitario y el alumnado escolar desarrollando las creaciones artísticas colaborativas. El grupo de discusión se llevó a cabo en una sesión de dos horas de duración.

Este estudio se ha realizado de conformidad con las recomendaciones del Comité de Ética de la Investigación de la Universidad de las Islas Baleares (autorización nº 95CER18).

\subsection{Puesta en práctica de la acción}

La acción pedagógica 'Sonorización del Barrio: el Latido Sonoro’ fue diseñada a partir de cuatro características: vinculación con el medio, trabajo cooperativo y colaborativo, enfoque creativo y atención a la dimensión social, como se explica a continuación:

Una característica clave de la acción desarrollada fue la vinculación con el medio, al plantearse como una práctica situada, lo que resuena en los argumentos de Salvà-Tomàs, JaumeAndrover y Berbel-Gómez (2019) que apuestan por dotar al futuro docente de experiencias de aprendizaje situadas basadas en contextos reales que permitan desarrollar comunidades

\footnotetext{
${ }^{1}$ Mediante el uso de diferentes aplicaciones con dispositivos móviles, el mapeo consistió en incluir la mayor información posible sobre las localizaciones seleccionadas: coordenadas de latitud y longitud (GPS), fotografías panorámicas del lugar, grabaciones de audio con información sobre el tiempo de duración, fecha y hora exacta, así como otros datos que pudieran ser de interés en relación con el paisaje sonoro registrado.
}

@ Noemy Berbel Gómez, Adolf Murillo Ribes y Mª Elena Riaño Galán. The content of this article is the sole responsibility of the authors. The Revista Electrónica de LEEME and Universitat de València are not liable for any legal actions that may arise involving the article's content. Revista Electrónica de LEEME - Lista Electrónica Europea de Música en la Educación-. http://ojs.uv.es/index/php/LEEME/index ISSN: 1575-9563. Editores: Universidad de Valencia y Jesús Tejada. Visibilidad de esta revista: SCOPUS, Emerging Sources Citation Index (Clarivate), EBSCO, CINDOC (CSIC), Citefactor, COPAC, Dialnet, DICE (CSIC), DOAJ, e-revistas (CSIC), EBSCO Premier, ERIH+, Gale Cengage Learning, IN-RECS, IRESIE, LATINDEX, MIAR, OCLC Worldcat, RESH, REDIB, RILM Core Journals, SUDOC, ULRICHS. Esta revista es de acceso libre mediante licencia Creative Commons 4.0 CC by. Política de archivado: etiqueta verde SHERPA-ROMEO. 


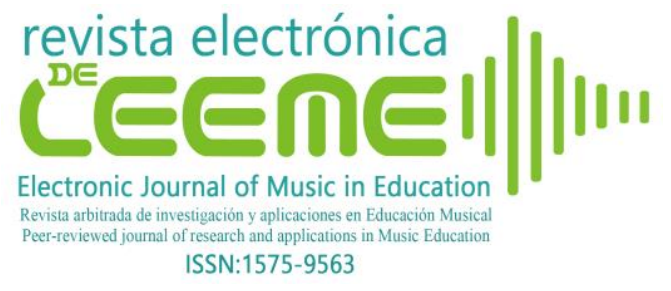

Noemy Berbel Gómez, Adolf Murillo Ribes y Mª Elena Riaño Galán Cuando el barrio educa: aprendizaje situado y creación artística colaborativa como herramienta en la formación musical (Revista Electrónica de LEEME) Número 46, pp. 68-91 https://ojs.uv.es/index.php/LEEME/index ISSN:1575-9563

discursivas de aprendizaje (Robinson, 2016). La materia prima de la que se partió fue el material sonoro que brindó el propio barrio, a partir de su exploración. La captura e intervención sonora sobre el espacio público permitió, por un lado, aproximarse al paisaje sonoro del barrio y, por otro, conectar con el entorno próximo del infante, coincidiendo con las ideas de Bartolomé (2017) y Haston y Russell (2012), en las que el alumnado universitario fue capaz de llegar a comprender la realidad del territorio y las necesidades y problemas de sus habitantes, además de desarrollar conocimientos pedagógicos generales y específicos.

Otra importante característica del diseño pedagógico de la acción requería que las diversas actividades fueran fruto de un trabajo cooperativo y colaborativo entre el alumnado universitario y el de Primaria, con la participación de músicos/expertos dinamizadores de la propuesta. La guía por parte de especialistas o expertos sirvió como una preparación práctica exhaustiva para los profesores generalistas en formación que implicaba "autodesarrollo colaborativo" (Barret, Zhukov y Welch, 2019).

Una tercera característica planificada se relacionó con el modo de abordar el desarrollo de la propuesta desde un enfoque creativo a partir de la escucha atenta y la experimentación sonora a través de las intervenciones sobre el mobiliario del barrio. Estas, posteriormente, se integrarían en una composición musical propia. Este enfoque creativo se amplió en la propuesta a través de la hibridación de lenguajes, donde tomó parte todo el cuerpo en el proceso de aprender (Hutchins, 1995; Leman, 2007).

Finalmente, la última y cuarta característica fue atender a la dimensión social, al introducir las realidades y problemáticas del contexto en la propuesta con un fin concienciador capaz de generar vínculos (Doberneck et al., 2010) y, a la vez, transformador (O’Neill, 2014), desde un enfoque artístico. En la acción desarrollada se trataron problemáticas existentes en el barrio, como multiculturalidad, falta de cohesión social, drogas, violencia, gentrificación ${ }^{2}$ o basura. Se propusieron acciones artísticas que pretendieron no solo mostrar la cara más dura de la realidad, sino que buscaban la toma de conciencia desde el respeto. Asimismo, se aportaron soluciones basadas en la colaboración entre todos los participantes, canalizadas a través de la expresión y la acción artística. Se describe a continuación las actividades desarrolladas en los dos talleres.

\footnotetext{
${ }^{2}$ Gentrificación es una adaptación adecuada al español del término inglés gentrification, con el que se alude al proceso mediante el cual la población original de un sector o barrio, generalmente céntrico y popular, es progresivamente desplazada por otra de un nivel adquisitivo mayor.
}

@ Noemy Berbel Gómez, Adolf Murillo Ribes y Mª Elena Riaño Galán. The content of this article is the sole responsibility of the authors. The Revista Electrónica de LEEME and Universitat de València are not liable for any legal actions that may arise involving the article's content. Revista Electrónica de LEEME - Lista Electrónica Europea de Música en la Educación-. http://ojs.uv.es/index/php/LEEME/index ISSN: 1575-9563. Editores: Universidad de Valencia y Jesús Tejada. Visibilidad de esta revista: SCOPUS, Emerging Sources Citation Index (Clarivate), EBSCO, CINDOC (CSIC), Citefactor, COPAC, Dialnet, DICE (CSIC), DOAJ, e-revistas (CSIC), EBSCO Premier, ERIH+, Gale Cengage Learning, IN-RECS, IRESIE, LATINDEX, MIAR, OCLC Worldcat, RESH, REDIB, RILM Core Journals, SUDOC, ULRICHS. Esta revista es de acceso libre mediante licencia Creative Commons 4.0 CC by. Política de archivado: etiqueta verde SHERPA-ROMEO. 


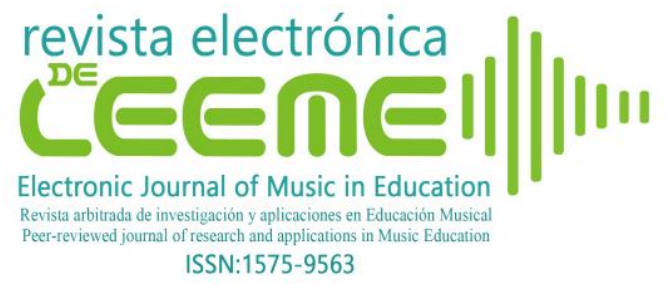

Noemy Berbel Gómez, Adolf Murillo Ribes y Mª Elena Riaño Galán Cuando el barrio educa: aprendizaje situado y creación artística colaborativa como herramienta en la formación musical (Revista Electrónica de LEEME) Número 46, pp. 68-91 https://ojs.uv.es/index.php/LEEME/index ISSN:1575-9563

\subsubsection{Taller 1 'Escuchar el Barrio: Captura Sonora e Intervención en el Espacio'}

Fue diseñado teniendo en cuenta las posibilidades que un mismo espacio ofrece, por un lado, como un paisaje sonoro en sí mismo y, por otro, como un lugar que puede ser intervenido creativamente. Las actividades propuestas fueron:

Captura Sonora: Se realizó un registro sonoro del barrio a partir de algunos puntos de interés seleccionados y visitados previamente, como, por ejemplo, la Fábrica Ribas actualmente abandonada o el Museo Krekovic, entrevistando, además, a algunos habitantes del barrio. Se exploraron auditivamente localizaciones específicas de acuerdo con unas pautas de escucha dadas y basadas en parámetros sonoros tales como intensidad, duración, diversidad tímbrica, altura o localización, entre otros, con el propósito de realizar una escucha atenta e identificar cómo suena el barrio en la actualidad. Esta actividad fue realizada de forma conjunta por el alumnado universitarios y el alumnado de Primaria.

Intervención Sonora en el Espacio: Consistió en realizar una performance en los mismos espacios seleccionados para la actividad anterior. Se intervinieron los puntos de interés citados a través de la interacción con los materiales urbanos y elementos arquitectónicos de cada lugar, desde la improvisación, la escucha colectiva y la experimentación sonora. Posteriormente, se crearon intervenciones sonoras en el espacio a partir de dicha experimentación (improvisación libre), llevándose a cabo una performance en los espacios de origen de los sonidos capturados. Esta actividad fue realizada de forma conjunta por el alumnado universitario y el alumnado de Educación Primaria.

A continuación, se presenta una breve descripción del alumnado universitario como ejemplo de una de las intervenciones sonoras. La estudiante 2 afirmó:

\footnotetext{
"escuchamos de fondo un rozamiento constante de un objeto sobre una tubería de la fábrica, seguido por un golpe y un chasquido. Este conjunto de sonidos puede simbolizar el momento en el cual las máquinas de la fabrica estaban en funcionamiento. Para conseguir estos sonidos se usaron las manos y un objeto para golpear y otro para frotar"3.
}

\footnotetext{
${ }^{3}$ Un ejemplo de intervención sonora en la Fábrica Gorila se puede encontrar en: https://soundcloud.com/guillermopetricorena/gorila-is1
}

@ Noemy Berbel Gómez, Adolf Murillo Ribes y Mª Elena Riaño Galán. The content of this article is the sole responsibility of the authors. The Revista Electrónica de LEEME and Universitat de València are not liable for any legal actions that may arise involving the article's content. Revista Electrónica de LEEME - Lista Electrónica Europea de Música en la Educación-. http://ojs.uv.es/index/php/LEEME/index ISSN: 1575-9563. Editores: Universidad de Valencia y Jesús Tejada. Visibilidad de esta revista: SCOPUS, Emerging Sources Citation Index (Clarivate), EBSCO, CINDOC (CSIC), Citefactor, COPAC, Dialnet, DICE (CSIC), DOAJ, e-revistas (CSIC), EBSCO Premier, ERIH+, Gale Cengage Learning, IN-RECS, IRESIE, LATINDEX, MIAR, OCLC Worldcat, RESH, REDIB, RILM Core Journals, SUDOC, ULRICHS. Esta revista es de acceso libre mediante licencia Creative Commons 4.0 CC by. Política de archivado: etiqueta verde SHERPA-ROMEO. 


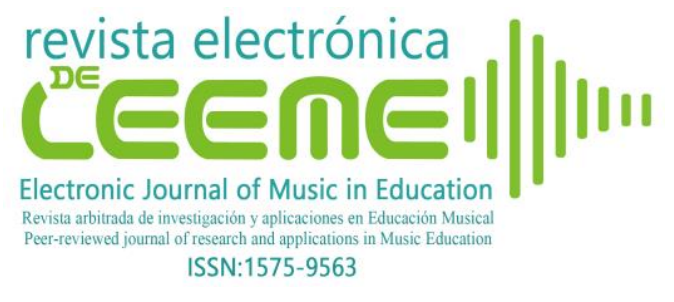

Noemy Berbel Gómez, Adolf Murillo Ribes y Mª Elena Riaño Galán Cuando el barrio educa: aprendizaje situado y creación artística colaborativa como herramienta en la formación musical (Revista Electrónica de LEEME) Número 46, pp. 68-91 https://ojs.uv.es/index.php/LEEME/index ISSN:1575-9563
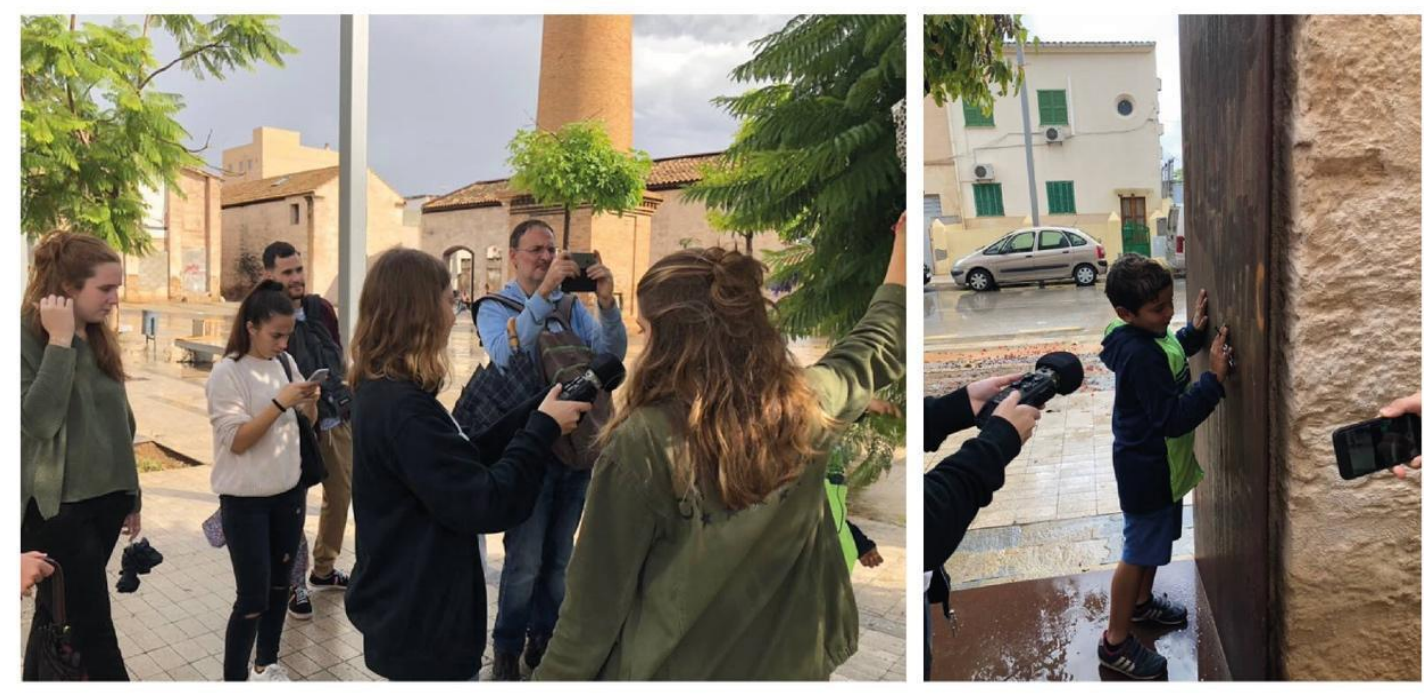

Figura 1. Captura e intervención sonora en el barrio

Composición musical: Tomando como referencia el material sonoro recogido en la captura e intervención, se realizaron creaciones musicales de forma colaborativa en las diferentes localizaciones del Barrio en las que se utilizaron herramientas tecnológicas. Estas composiciones sirvieron de material para llevar a cabo el Taller 2. Esta actividad fue realizada por el alumnado universitario ${ }^{4}$.

\subsubsection{Taller 2 'Cápsulas Creativas: Trazos en Corto-Mosaicos Humanos'}

Se diseñó para concienciar y generar conexiones ante las problemáticas del barrio haciendo uso de los materiales elaborados en el primer taller. Algunas de estas problemáticas (multiculturalidad, falta de cohesión social, drogas, violencia, gentrificación o basura), fueron detectadas a partir de la indagación del alumnado universitario en el contexto del barrio. Posteriormente, se realizaron propuestas creativas multidisciplinares desde la hibridación de lenguajes musical, corporal y plástico-visual. Las dos actividades propuestas fueron:

Trazos en Corto: Se presentó un material consistente en un juego de tarjetas con pequeñas partituras gráficas y seis opciones de interpretación aleatorias -que correspondían a las diferentes maneras de interpretar dichas grafías (cuerpo, voz, instrumentos, electrónica, objetos y movimiento)-. El fin era generar una primera experimentación sonora utilizando las grafías

\footnotetext{
4 Ejemplo de composición sonora elaborada a partir de la intervención en el Parque Krekovic: https://mega.nz/file/MTBB2Qpb\#Mu2Q FzrgtcHs1y4hOlUH83li0w2vkypAACXADIkO6A
}

@ Noemy Berbel Gómez, Adolf Murillo Ribes y Mª Elena Riaño Galán. The content of this article is the sole responsibility of the authors. The Revista Electrónica de LEEME and Universitat de València are not liable for any legal actions that may arise involving the article's content. Revista Electrónica de LEEME - Lista Electrónica Europea de Música en la Educación-. http://ojs.uv.es/index/php/LEEME/index ISSN: 1575-9563. Editores: Universidad de Valencia y Jesús Tejada. Visibilidad de esta revista: SCOPUS, Emerging Sources Citation Index (Clarivate), EBSCO, CINDOC (CSIC), Citefactor, COPAC, Dialnet, DICE (CSIC), DOAJ, e-revistas (CSIC), EBSCO Premier, ERIH+, Gale Cengage Learning, IN-RECS, IRESIE, LATINDEX, MIAR, OCLC Worldcat, RESH, REDIB, RILM Core Journals, SUDOC, ULRICHS. Esta revista es de acceso libre mediante licencia Creative Commons 4.0 CC by. Política de archivado: etiqueta verde SHERPA-ROMEO. 


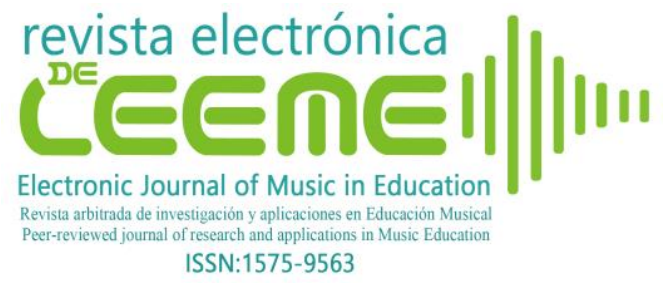

Noemy Berbel Gómez, Adolf Murillo Ribes y Mª Elena Riaño Galán Cuando el barrio educa: aprendizaje situado y creación artística colaborativa como herramienta en la formación musical (Revista Electrónica de LEEME) Número 46, pp. 68-91 https://ojs.uv.es/index.php/LEEME/index

ISSN:1575-9563

contemporáneas como detonante de partida. Durante un tiempo asignado, cada grupo ensayó su interpretación y la presentó públicamente ante sus compañeros. Esta actividad fue realizada por alumnado universitario y de Educación Primaria.

Mosaicos Humanos: A partir de la experiencia de la actividad 'Trazos en Corto' junto con las composiciones musicales realizadas con el material recogido en el Taller 1 ('Escuchar el Barrio'), se realizaron varias propuestas performativas, utilizando el cuerpo y el movimiento. Todas las propuestas introdujeron las diferentes problemáticas o realidades del barrio detectadas $^{5}$. Esta actividad fue realizada por el profesorado en formación y el alumnado de Educación Primaria.
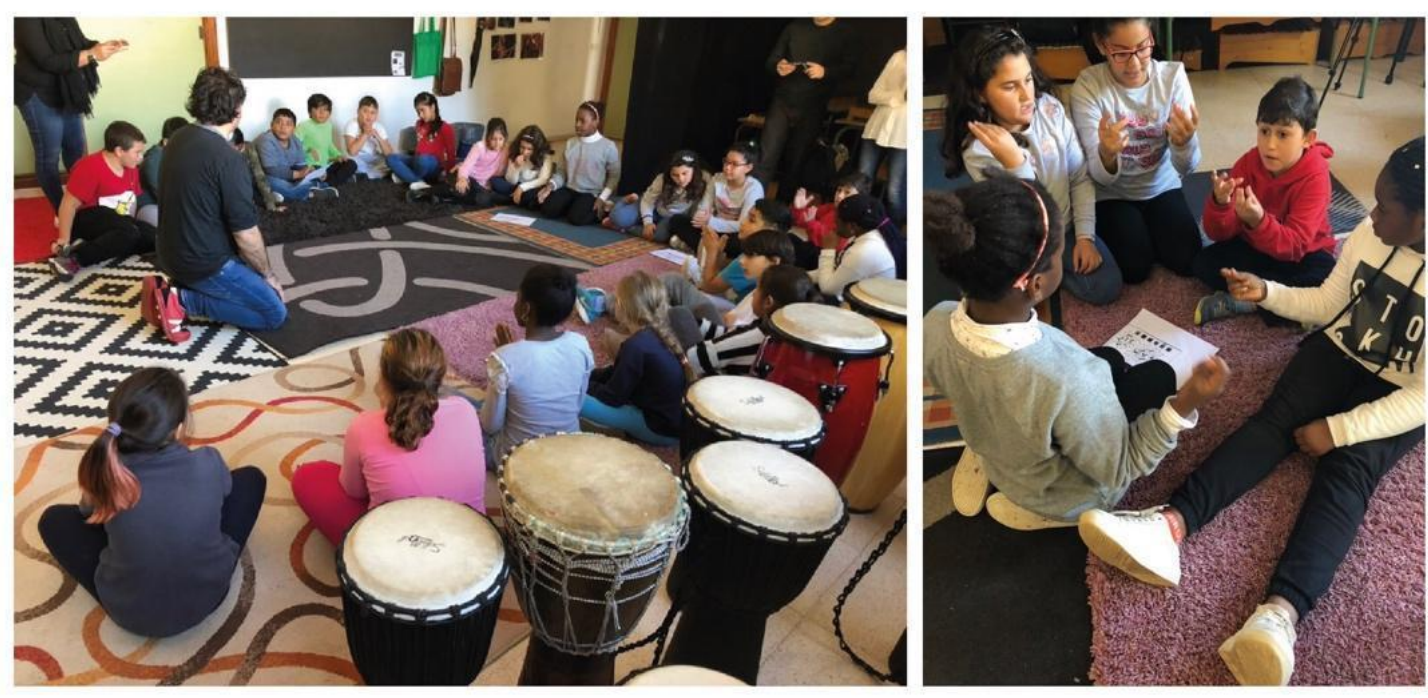

Figura 2. 'Trazos en Corto' trabajo grupo-clase alumnado de Educación Primaria con notación gráfica

\footnotetext{
${ }^{5}$ Ejemplo de 'Mosaico Humano' - Fábrica Ribas: https://youtu.be/TTtMv5MX-vE
}

@ Noemy Berbel Gómez, Adolf Murillo Ribes y Mª Elena Riaño Galán. The content of this article is the sole responsibility of the authors. The Revista Electrónica de LEEME and Universitat de València are not liable for any legal actions that may arise involving the article's content. Revista Electrónica de LEEME - Lista Electrónica Europea de Música en la Educación-. http://ojs.uv.es/index/php/LEEME/index ISSN: 1575-9563. Editores: Universidad de Valencia y Jesús Tejada. Visibilidad de esta revista: SCOPUS, Emerging Sources Citation Index (Clarivate), EBSCO, CINDOC (CSIC), Citefactor, COPAC, Dialnet, DICE (CSIC), DOAJ, e-revistas (CSIC), EBSCO Premier, ERIH+, Gale Cengage Learning, IN-RECS, IRESIE, LATINDEX, MIAR, OCLC Worldcat, RESH, REDIB, RILM Core Journals, SUDOC, ULRICHS. Esta revista es de acceso libre mediante licencia Creative Commons 4.0 CC by. Política de archivado: etiqueta verde SHERPA-ROMEO. 


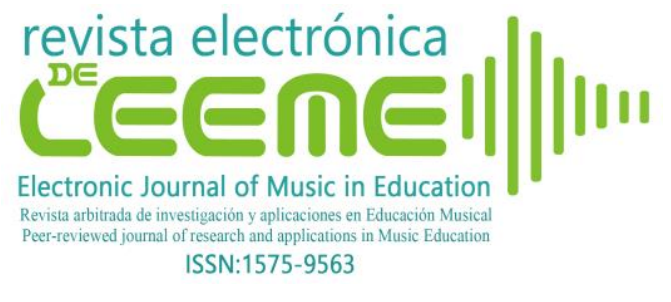

Noemy Berbel Gómez, Adolf Murillo Ribes y Ma Elena Riaño Galán Cuando el barrio educa: aprendizaje situado y creación artística colaborativa como herramienta en la formación musical (Revista Electrónica de LEEME) Número 46, pp. 68-91 https://ojs.uv.es/index.php/LEEME/index ISSN:1575-9563

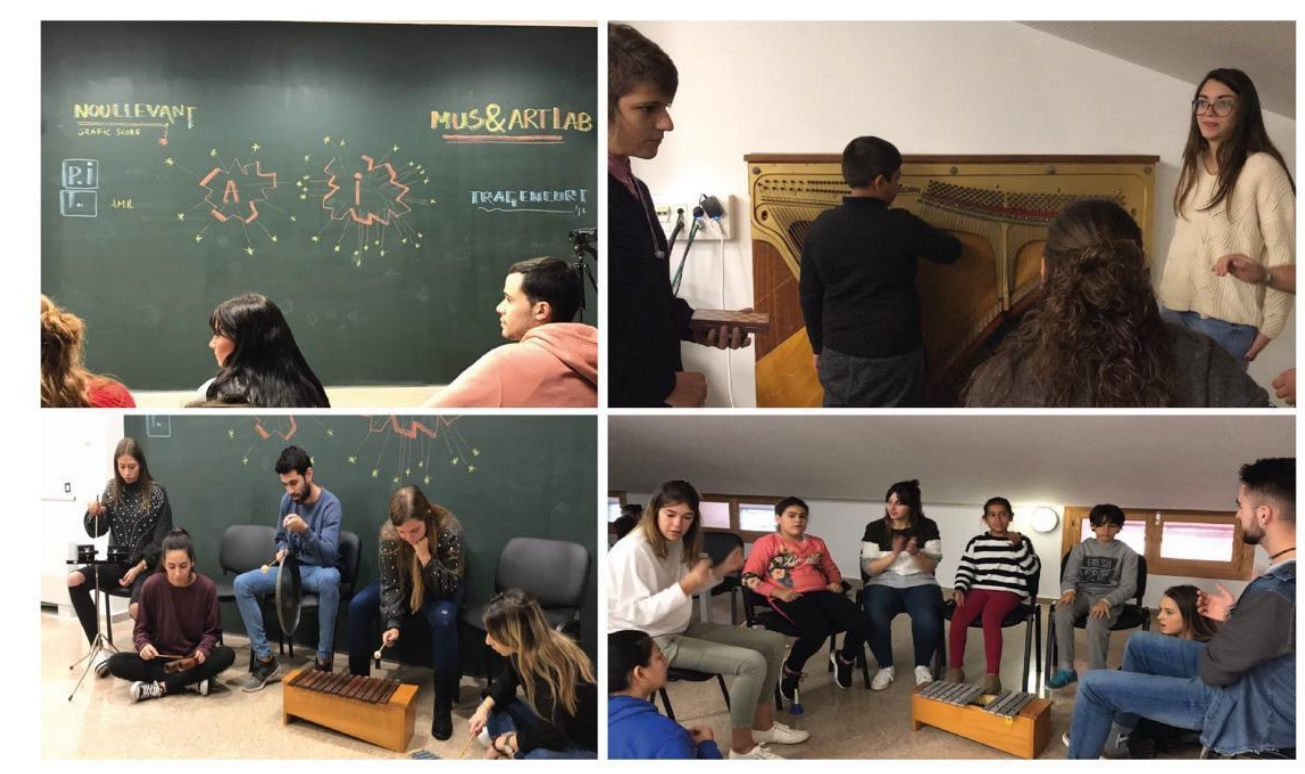

Figura 3. 'Mosaicos Humanos' trabajo colaborativo alumnado universidad y alumnado de Educación Primaria en el Mus\&Art LAB.

\section{Resultados}

Como se mencionó anteriormente, el objetivo de este estudio ha sido conocer las percepciones del profesorado generalista en formación inicial sobre la relevancia de la acción en que participaron.

Se estableció un grupo de discusión sobre el proceso experimentado conformado por la totalidad del alumnado participante en la acción pedagógica. Emergieron tres dimensiones a partir del análisis de las transcripciones del grupo de discusión (Dey, 2005): 1) "Dimensión metodológica": valor de compartir, nuevos enfoques metodológicos, la importancia del entorno y de la diversidad cultural, perspectivas futuras; 2) "Dimensión creativo/musical": espacios de conocimiento con el otro y colaboración, gestión de los grupos, el tiempo: un factor a tener en cuenta, ampliar la mirada; 3) "Dimensión Social": resolución de conflictos o situaciones personales del alumnado de Primaria, la colaboración con el otro, el valor de lo diverso y diferente, arte y transformación.

@ Noemy Berbel Gómez, Adolf Murillo Ribes y Mª Elena Riaño Galán. The content of this article is the sole responsibility of the authors. The Revista Electrónica de LEEME and Universitat de València are not liable for any legal actions that may arise involving the article's content. Revista Electrónica de LEEME - Lista Electrónica Europea de Música en la Educación-. http://ojs.uv.es/index/php/LEEME/index ISSN: 1575-9563. Editores: Universidad de Valencia y Jesús Tejada. Visibilidad de esta revista: SCOPUS, Emerging Sources Citation Index (Clarivate), EBSCO, CINDOC (CSIC), Citefactor, COPAC, Dialnet, DICE (CSIC), DOAJ, e-revistas (CSIC), EBSCO Premier, ERIH+, Gale Cengage Learning, IN-RECS, IRESIE, LATINDEX, MIAR, OCLC Worldcat, RESH, REDIB, RILM Core Journals, SUDOC, ULRICHS. Esta revista es de acceso libre mediante licencia Creative Commons 4.0 CC by. Política de archivado: etiqueta verde SHERPA-ROMEO. 


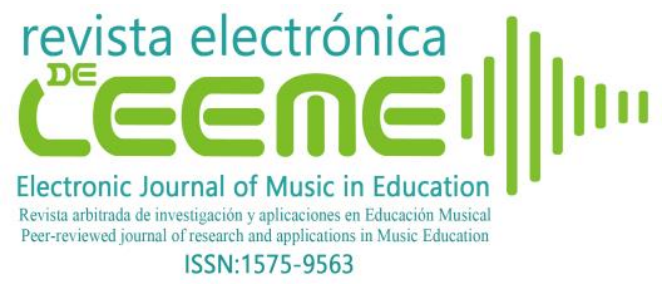

Noemy Berbel Gómez, Adolf Murillo Ribes y Mª Elena Riaño Galán Cuando el barrio educa: aprendizaje situado y creación artística colaborativa como herramienta en la formación musical (Revista Electrónica de LEEME) Número 46, pp. 68-91 https://ojs.uv.es/index.php/LEEME/index SSN:1575-9563

\title{
4.1 Dimensión metodológica
}

\section{El valor del compartir}

La mayoría de los participantes hizo alusión a la importancia de poder contactar con los niños y trabajar en una acción conjunta que les supuso una motivación para continuar formándose como docentes. La estudiante 25 señaló que "la experiencia se agradece mucho porque es el primer contacto con los niños en tercer curso". De manera similar, el estudiante 3 expresó:

\begin{abstract}
"Poder experimentar de primera mano con niños a los cuales educaré en un futuro me da ganas de seguir formándome para dedicarme a esta profesión. Me ha aportado una dosis de práctica con alumnado, una dosis de motivación para seguir el curso, conocimiento musical e ideas para la creación y composición".
\end{abstract}

\section{Nuevos enfoques metodológicos}

Los participantes valoraron que la metodología utilizada se alejaba de la rigidez y los convencionalismos aceptando la innovación como un elemento importante en la profesión docente, como señaló el estudiante 4: "siempre se debe adaptar la metodología de enseñanza al alumnado, es bueno innovar y experimentar nuevas sensaciones y tener nuevas experiencias". La estudiante 28 mencionó haber conocido nuevas metodologías de trabajo, destacando: "las diferentes maneras en las que se puede aplicar la música en el ámbito escolar saliendo de las clases tradicionales". Esto fue reseñado igualmente por el estudiante 12: "innovar, creo que es un aspecto fundamental a la hora de ser docente. El hecho de proponer nuevos proyectos siempre puede llevar a cabo una mejora y, sobre todo, motivación y ganas de aprender por parte de los niños". Asimismo, incidieron en la importancia de crear entornos abiertos de aprendizaje donde se posibilite el desarrollo de la creatividad al afirmar que: "dar libertad para trabajar la creatividad es un aspecto muy importante y no se trabaja lo suficiente en las aulas" (estudiante 43).

\section{La importancia del entorno y de la diversidad cultural}

Muchas respuestas coincidieron en otorgar importancia al conocimiento del entorno, el cual permite llegar a un mejor entendimiento de las realidades para poder buscar soluciones desde la participación e implicación de todos los actores. En opinión de la estudiante 40:

"He aprendido formas de integrar o implicar a toda la comunidad educativa. Este proyecto nos ha hecho trabajar más el aspecto real de la educación, el poder salir del aula, el trabajar con alumnado, con docentes, la organización del centro, etc."

@ Noemy Berbel Gómez, Adolf Murillo Ribes y Mª Elena Riaño Galán. The content of this article is the sole responsibility of the authors. The Revista Electrónica de LEEME and Universitat de València are not liable for any legal actions that may arise involving the article's content. Revista Electrónica de LEEME - Lista Electrónica Europea de Música en la Educación-. http://ojs.uv.es/index/php/LEEME/index ISSN: 1575-9563. Editores: Universidad de Valencia y Jesús Tejada. Visibilidad de esta revista: SCOPUS, Emerging Sources Citation Index (Clarivate), EBSCO, CINDOC (CSIC), Citefactor, COPAC, Dialnet, DICE (CSIC), DOAJ, e-revistas (CSIC), EBSCO Premier, ERIH+, Gale Cengage Learning, IN-RECS, IRESIE, LATINDEX, MIAR, OCLC Worldcat, RESH, REDIB, RILM Core Journals, SUDOC, ULRICHS. Esta revista es de acceso libre mediante licencia Creative Commons $4.0 \mathrm{CC}$ by. Política de archivado: etiqueta verde SHERPA-ROMEO. 


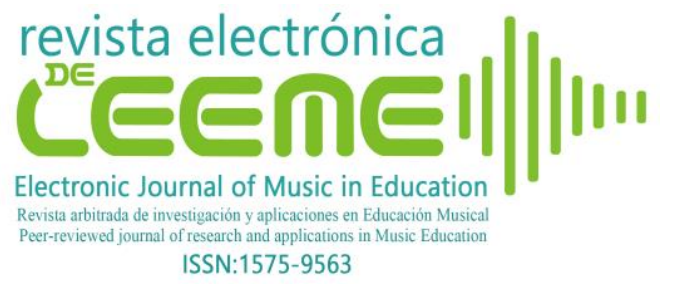

Noemy Berbel Gómez, Adolf Murillo Ribes y Mª Elena Riaño Galán Cuando el barrio educa: aprendizaje situado y creación artística colaborativa como herramienta en la formación musical (Revista Electrónica de LEEME) Número 46, pp. 68-91 https://ojs.uv.es/index.php/LEEME/index

ISSN:1575-9563

En esta misma línea, el estudiante 14 mencionó que se había "llevado a cabo un proyecto interesante para conocer un contexto real, con alumnado y situaciones muy diversas. En una asignatura sin salir del aula y sin ese contacto, no se aprende de la misma manera".

\section{Perspectivas futuras}

Tres estudiantes opinaron que participar en la experiencia cambió su percepción y aumentó su confianza para hacer uso de este tipo de enfoques en su trabajo como docentes. En este sentido el estudiante1 expresó: "participar en el proyecto me ha dotado de capacidades para discriminar el potencial artístico de espacios y objetos no convencionales"; el estudiante 21 afirmó: "ha aumentado mi interés y he aprendido que experiencias como esta pueden ser de gran utilidad para trabajar con niños de Educación Primaria". Y para la estudiante 19: "el arte puede usarse para desarrollar el currículum de manera transversal con otras asignaturas. Me planteo desarrollarlas en el futuro".

\subsection{Dimensión creativo/musical}

\section{Espacios de conocimiento con el otro y colaboración}

Se destacó la oportunidad de trabajar con infantes de forma más libre dejando espacio para crear y explorar a partir de los recursos del barrio, como es el caso del estudiante 11: "el momento que más me ha gustado es cuando hemos ido a nuestro punto de interés (la iglesia) y poder estar con los niños y grabar los sonidos; es cuando hemos tenido más tiempo para la creatividad". La estudiante 2 afirmó:

\footnotetext{
"Con los niños pudimos hacer que nuestras ideas volaran, podíamos hacer lo que nosotros quisiéramos, coger los sonidos que quisiéramos. Los niños disfrutaban jugando con el agua, dando golpecitos con la piedrecita. Es lo que realmente más me ha gustado, ha sido donde de verdad hemos podido crear".
}

\section{Gestión de los grupos}

Las dinámicas que se generaron en los procesos creativos colaborativos fueron un elemento de preocupación del alumnado: "cuando te dan la libertad para crear, da miedo" (estudiante 47). "No hay que forzar al alumnado, pero es verdad que hay que tener en cuenta esa situación en la que improvisas y la vergüenza se te va quitando. La música facilita eso, estas actividades" (estudiante 50). 


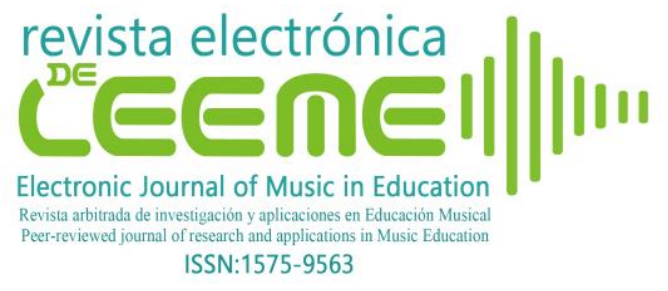

Noemy Berbel Gómez, Adolf Murillo Ribes y Mª Elena Riaño Galán Cuando el barrio educa: aprendizaje situado y creación artística colaborativa como herramienta en la formación musical (Revista Electrónica de LEEME) Número 46, pp. 68-91 https://ojs.uv.es/index.php/LEEME/index ISSN:1575-9563

\section{Importancia del factor tiempo}

Un mayor tiempo para la exploración, sin un horario estricto, puede facilitar los procesos reflexivos y la creatividad. En esta experiencia se dedicó una mañana lectiva completa para que cada grupo de trabajo gestionara el tiempo de manera flexible y autónoma. Algunos estudiantes sintieron cómo las propuestas creativas reclaman una nueva disposición de la gestión del tiempo a la hora de favorecer espacios de libre creación. La estudiante 21 comentó, al respecto, que "en propuestas creativas como esta, el tiempo ha condicionado de forma positiva a los niños".

\section{Ampliar la mirada}

Varios participantes opinaron que esta forma de abordar las artes aporta nuevas posibilidades para transformar y hacer uso de los sonidos cotidianos de una forma más creativa, asumiendo el riesgo desde una apertura continua a la novedad: "no creía que podría ser capaz de crear composiciones musicales creativas a partir de captaciones o intervenciones creadas por nosotras mismas, y lo hemos llevado a cabo" (estudiante 15).

Ampliar la mirada también implica potenciar nuevas escuchas y aprender a descubrir nuevas posibilidades en el entorno. La estudiante 16 comentó: "he aprendido a prestar más atención a todo aquel sonido que nos rodea, experimentar a través de la creatividad de una misma y de sus compañeras y, sobretodo, crear y componer. La estudiante 44 dijo "que pueden salir creaciones artísticas a partir de objetos o lugares que nunca, antes, hubiera imaginado".

\subsection{Dimensión social}

\section{Resolución de conflictos o situaciones personales del alumnado de Primaria}

La acción artística sirvió como reflexión para un gran número de participantes cuando han de resolverse conflictos. En este caso, permitió situar al estudiante ante las problemáticas del barrio a través de las voces de los niños y sus vivencias personales. Para el estudiante 61: "en la carrera no te enseñan cómo debemos actuar cuando te enfrentas delante de un problema de cómo se sienten los niños en casa, un problema fuera de la escuela. Recursos como este nos pueden ser útiles". Otro ejemplo lo aporta el estudiante 63:

"Es muy importante conocer el barrio donde está el colegio para poder llegar a entender e involucrar al alumnado, el conocimiento de las diferentes situaciones personales en las que se pueden encontrar los niños y la necesidad de buscar una solución a través de didácticas artísticas".

@ Noemy Berbel Gómez, Adolf Murillo Ribes y Mª Elena Riaño Galán. The content of this article is the sole responsibility of the authors. The Revista Electrónica de LEEME and Universitat de València are not liable for any legal actions that may arise involving the article's content. Revista Electrónica de LEEME - Lista Electrónica Europea de Música en la Educación-. http://ojs.uv.es/index/php/LEEME/index ISSN: 1575-9563. Editores: Universidad de Valencia y Jesús Tejada. Visibilidad de esta revista: SCOPUS, Emerging Sources Citation Index (Clarivate), EBSCO, CINDOC (CSIC), Citefactor, COPAC, Dialnet, DICE (CSIC), DOAJ, e-revistas (CSIC), EBSCO Premier, ERIH+, Gale Cengage Learning, IN-RECS, IRESIE, LATINDEX, MIAR, OCLC Worldcat, RESH, REDIB, RILM Core Journals, SUDOC, ULRICHS. Esta revista es de acceso libre mediante licencia Creative Commons 4.0 CC by. Política de archivado: etiqueta verde SHERPA-ROMEO. 


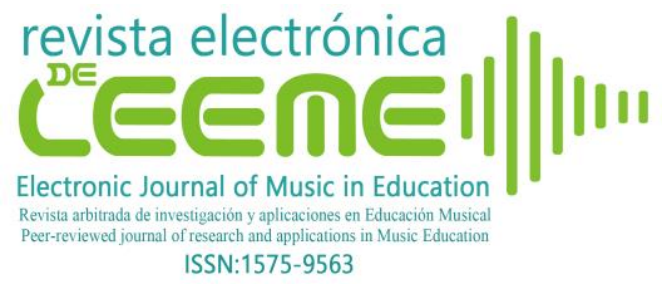

Noemy Berbel Gómez, Adolf Murillo Ribes y Mª Elena Riaño Galán Cuando el barrio educa: aprendizaje situado y creación artística colaborativa como herramienta en la formación musical (Revista Electrónica de LEEME) Número 46, pp. 68-91 https://ojs.uv.es/index.php/LEEME/index ISSN:1575-9563

\title{
La colaboración con el otro
}

Un aspecto que valoró la mayoría de los estudiantes fue la necesaria implicación de todos los agentes educativos para que verdaderamente tenga lugar el proceso de cambio, empezando desde la escuela. El estudiante 13 manifestó que

\begin{abstract}
"es necesaria la participación de todo el centro educativo y de las familias para llevar a cabo proyectos como este"; del mismo modo el estudiante 30 afirmó que "el cambio debe empezar con el alumnado realizando proyectos de este tipo ya que se sienten implicados, creando así un espíritu crítico, provocando así un cambio".
\end{abstract}

\section{El valor de lo diverso y diferente}

Los estudiantes reconocieron en la diversidad una oportunidad para observar con una mirada más social y profunda, así como desprenderse de ciertos estereotipos. El estudiante 14 afirmó que "hemos podido ver más allá de lo que vemos a simple vista, nos hemos quitado algunos prejuicios y aprendido de otras personas y culturas." El alumnado fue consciente de la necesidad de indagar en dichos aspectos; en opinión del estudiante 55: "hay que tener en cuenta la diversidad multicultural, nuestros orígenes y tradiciones y, sobre todo, donde residimos". Del mismo modo, el estudiante 64 argumentó que: "el conocer diversos entornos te hace aprender de diferentes puntos de vista y realidades. Hay que tener en cuenta que existen muchas perspectivas de una realidad común y tan solo hay que conocerlas para poder cambiar de punto de vista”.

\section{Arte y transformación}

La mayoría de las opiniones valoraron el arte como una vía de acceso al conocimiento de los problemas de la realidad actual y como herramienta que puede transformar la realidad social. El estudiante 30 señaló al respecto que:

"El arte es una forma de expresar diferentes sentimientos de manera universal porque cualquier expresión artística puede hacerte ver la realidad. En este caso, a través del arte hemos visto las problemáticas del barrio. Nos ha ofrecido la posibilidad de conocer más allá.”

Las producciones artísticas resultantes, como se ha ejemplificado, constituyen una herramienta que también ha permitido al alumnado universitario ampliar su conocimiento a nivel social, como afirma la estudiante 32: "las prácticas artísticas me han hecho ver una parte de la sociedad que desconocía, alguna de las características de nuevas culturas y formas de vivir diferentes a la mía" o el estudiante 7: "las prácticas artísticas pueden servir de medio de

@ Noemy Berbel Gómez, Adolf Murillo Ribes y Mª Elena Riaño Galán. The content of this article is the sole responsibility of the authors. The Revista Electrónica de LEEME and Universitat de València are not liable for any legal actions that may arise involving the article's content. Revista Electrónica de LEEME - Lista Electrónica Europea de Música en la Educación-. http://ojs.uv.es/index/php/LEEME/index ISSN: 1575-9563. Editores: Universidad de Valencia y Jesús Tejada. Visibilidad de esta revista: SCOPUS, Emerging Sources Citation Index (Clarivate), EBSCO, CINDOC (CSIC), Citefactor, COPAC, Dialnet, DICE (CSIC), DOAJ, e-revistas (CSIC), EBSCO Premier, ERIH+, Gale Cengage Learning, IN-RECS, IRESIE, LATINDEX, MIAR, OCLC Worldcat, RESH, REDIB, RILM Core Journals, SUDOC, ULRICHS. Esta revista es de acceso libre mediante licencia Creative Commons 4.0 CC by. Política de archivado: etiqueta verde SHERPA-ROMEO. 


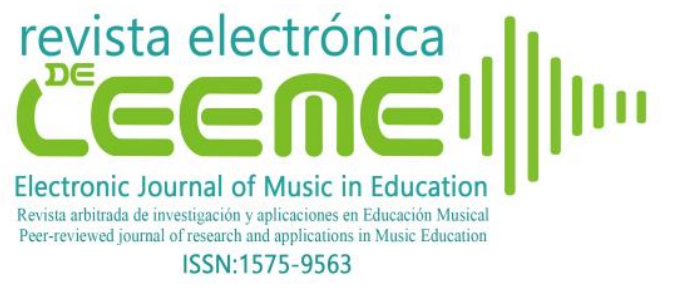

Noemy Berbel Gómez, Adolf Murillo Ribes y Mª Elena Riaño Galán Cuando el barrio educa: aprendizaje situado y creación artística colaborativa como herramienta en la formación musical (Revista Electrónica de LEEME) Número 46, pp. 68-91 https://ojs.uv.es/index.php/LEEME/index ISSN:1575-9563

expresión para mejorar los lugares más desfavorecidos. Se puede utilizar el arte para llegar a la sociedad".

\section{Discusión y conclusiones}

Es necesario dotar al futuro profesorado, tanto generalista como especialista de Música, de experiencias artísticas significativas, desde un enfoque creativo y situado (Wilson y McDonald, 2012). Sin embargo, en la práctica, este tipo de propuestas presentan ciertas dificultades para ser abordadas desde la formación inicial del docente (Harris y de Bruin, 2018). Por ello, cabe incidir en la relevancia de llevar a cabo estudios de corte pedagógico que ofrezcan a docentes y futuros docentes pautas para implementar propuestas en el aula que fomenten aprendizajes situados desde un enfoque creativo y colaborativo (Harris, 2014).

El objetivo de este estudio era conocer las percepciones de futuros docentes de Educación Primaria en el ámbito de la Educación Musical sobre la relevancia de una acción pedagógica en la que participaron, basada en la creación sonora colaborativa y contextualizada en una situación real.

En primer lugar, en relación con los aspectos metodológicos, el alumnado valoró de forma satisfactoria el trabajo conjunto con los niños y resultó un aspecto motivador. Sus opiniones positivas y el optimismo mostrado recuerdan a las ideas de Vries (2013) en relación con su autoeficacia y confianza para continuar su formación como docentes y desarrollar su futura labor profesional. En este mismo sentido, los participantes también destacaron la importancia de innovar y abordar nuevos enfoques metodológicos en el desarrollo de la profesión docente. El alumnado llevó a cabo una crítica a los modelos cerrados, direccionales, que no permiten una co-construcción del conocimiento en pro de unos aprendizajes más creativos y flexibles (Anderson y Campbell, 2011). En sus argumentaciones, valoraron que este tipo de experiencias les permitió reflexionar y posicionarse ante el modelo de escuela que desean y desafió al alumnado a cuestionarse cómo abordar la enseñanza de la música en las escuelas (Burnard, 2012). Por tanto, es necesario propiciar en los futuros docentes propuestas diferentes, ya que estos suelen tener la tendencia a replicar los modelos docentes que han vivido como alumnado (Regelski, 2005). Los discentes demostraron valorar la diversidad y el conocimiento del entorno, y comprender de manera más profunda las realidades de un grupo de infantes, más allá del espacio-aula. El futuro docente debe ser capaz de ajustar sus futuras propuestas educativas a las necesidades reales de su alumnado (O’Neill, 2016). Otro aspecto que destacar fue su cambio de percepción sobre la utilidad del trabajo colaborativo, que ahora es mucho más positiva y los ha animado a utilizarlo en su futura labor profesional (Christophersen, 2013).

@ Noemy Berbel Gómez, Adolf Murillo Ribes y Mª Elena Riaño Galán. The content of this article is the sole responsibility of the authors. The Revista Electrónica de LEEME and Universitat de València are not liable for any legal actions that may arise involving the article's content. Revista Electrónica de LEEME - Lista Electrónica Europea de Música en la Educación-. http://ojs.uv.es/index/php/LEEME/index ISSN: 1575-9563. Editores: Universidad de Valencia y Jesús Tejada. Visibilidad de esta revista: SCOPUS, Emerging Sources Citation Index (Clarivate), EBSCO, CINDOC (CSIC), Citefactor, COPAC, Dialnet, DICE (CSIC), DOAJ, e-revistas (CSIC), EBSCO Premier, ERIH+, Gale Cengage Learning, IN-RECS, IRESIE, LATINDEX, MIAR, OCLC Worldcat, RESH, REDIB, RILM Core Journals, SUDOC, ULRICHS. Esta revista es de acceso libre mediante licencia Creative Commons 4.0 CC by. Política de archivado: etiqueta verde SHERPA-ROMEO. 


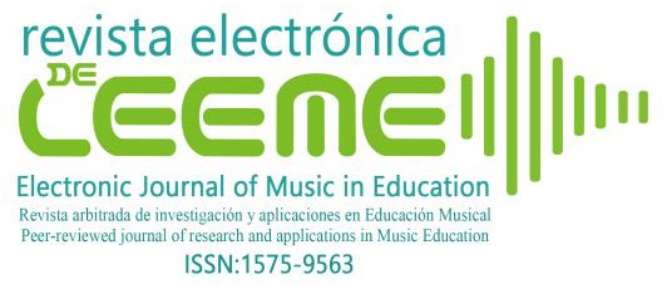

Noemy Berbel Gómez, Adolf Murillo Ribes y Mª Elena Riaño Galán Cuando el barrio educa: aprendizaje situado y creación artística colaborativa como herramienta en la formación musical (Revista Electrónica de LEEME) Número 46, pp. 68-91 https://ojs.uv.es/index.php/LEEME/index DOI: 10.7203/LEEME.46.17764

En segundo lugar, se puso énfasis en la dimensión creativo/musical de la acción. El alumnado destacó la importancia de una forma de creación libre, idea relacionada con el concepto de creatividad como "pensamiento de posibilidad" (Craft 2005). Algunos ejemplos de ello fueron: la libertad durante el proceso, la exploración a partir de los recursos del barrio, los momentos de descubrimiento o el aprendizaje no dirigido. La exploración implica buscar cambios, tomar riesgos y empujar a la experimentación (Marsh y Marsh, 2008; Marsh y Young, 2006). Es decir, las actividades propuestas estimularon la curiosidad del discente que, a su vez, estimuló su "necesidad de saber", en coherencia con los argumentos de Hickey (2012). Los participantes también manifestaron haber ampliado su percepción sobre las posibilidades creativas que ofrece el material sonoro que nos rodea, al estar en una posición más atenta y receptiva hacia cuestiones anteriormente no exploradas.

Esta idea está vinculada a las propuestas de Kenny (2015), quien defiende que las experiencias musicales creativas y de colaboración facilitan nuevos entendimientos de las pedagogías y prácticas de educación musical, desafiando sus ideas preconcebidas sobre la enseñanza de la música en las escuelas. Sin embargo, los participantes manifestaron preocupación ante las inseguridades provocadas por esa libertad concedida y las resistencias que genera enfrentarse a cosas nuevas. Los enfoques creativos adoptados en este proyecto permiten partir de la incertidumbre individual en un proceso en el que, posteriormente, al trabajar colaborativamente, se suma la colectividad de certezas individuales, las cuales se consolidan con el respaldo del grupo. Este tipo de propuestas ayudan a preparar al estudiante frente a la incertidumbre, lo inesperado, para lo que aún no se sabe (Elisondo et al. 2013), lo que posibilita incrementar su nivel de agencia ( $\left.\mathrm{O}^{\prime} \mathrm{Neill}, 2012\right)$ en cuanto a capacidad y habilidad para producir cambios considerados valiosos dentro de un entorno de oportunidades y restricciones (Hernández-Ponce, Rodríguez y Giménez, 2011). Otro aspecto demandado por el alumnado fue una dilatación en el tiempo que les permitiera una mayor exploración y flexibilidad en los procesos de libre creación. Es necesario proporcionar espacios y tiempos para la exploración para que se puedan abordar prácticas musicales creativas y compartidas. En estas prácticas, el tiempo dedicado a la exploración influirá en la calidad del resultado (Brinkman, 2010; Kenny, 2015).

En tercer lugar, los participantes identificaron la dimensión social de la propuesta, ya que confirmaron que este tipo de intervenciones les posibilita acercarse a la realidad del infante y tratar cuestiones o problemáticas, que no sabían cómo abordarlas previamente, a través de las didácticas. De acuerdo con Caeiro (2018), el ABC permite integrar y proyectar emociones, sentimientos e ideas a través de los lenguajes artísticos. Asimismo, se valoró el necesario trabajo en comunidad y la implicación de los diversos agentes educativos para que pueda darse un proceso transformador impulsado desde la escuela a través de proyectos de este tipo en los

@ Noemy Berbel Gómez, Adolf Murillo Ribes y Mª Elena Riaño Galán. The content of this article is the sole responsibility of the authors. The Revista Electrónica de LEEME and Universitat de València are not liable for any legal actions that may arise involving the article's content. Revista Electrónica de LEEME - Lista Electrónica Europea de Música en la Educación-. http://ojs.uv.es/index/php/LEEME/index ISSN: 1575-9563. Editores: Universidad de Valencia y Jesús Tejada. Visibilidad de esta revista: SCOPUS, Emerging Sources Citation Index (Clarivate), EBSCO, CINDOC (CSIC), Citefactor, COPAC, Dialnet, DICE (CSIC), DOAJ, e-revistas (CSIC), EBSCO Premier, ERIH+, Gale Cengage Learning, IN-RECS, IRESIE, LATINDEX, MIAR, OCLC Worldcat, RESH, REDIB, RILM Core Journals, SUDOC, ULRICHS. Esta revista es de acceso libre mediante licencia Creative Commons 4.0 CC by. Política de archivado: etiqueta verde SHERPA-ROMEO. 


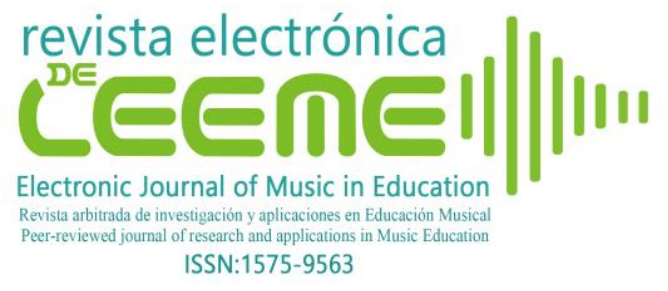

Noemy Berbel Gómez, Adolf Murillo Ribes y Mª Elena Riaño Galán Cuando el barrio educa: aprendizaje situado y creación artística colaborativa como herramienta en la formación musical (Revista Electrónica de LEEME) Número 46, pp. 68-91 https://ojs.uv.es/index.php/LEEME/index ISSN:1575-9563

que el alumnado se sienta implicado. A este respecto, Reynolds et al. (2005) afirman que en este tipo de experiencias reales los participantes pueden mostrar altos grados de motivación y conexión con la comunidad escolar. Es necesario por ello un compromiso de los docentes de música en crear asociaciones sólidas con las escuelas y las organizaciones comunitarias con una visión estratégica (Doberneck et al. 2010; Forrester, 2019). En la experiencia realizada, el alumnado reconoció que adentrarse en la diversidad del contexto y del discente de Primaria les posibilitó tener una oportunidad para observar con una mirada más social y profunda y a la vez desprenderse de ciertos estereotipos predefinidos. En este sentido, tal y como defiende Chou (2007), el profesorado en formación inicial suele mostrar muy poco conocimiento de los antecedentes interculturales y con limitadas experiencias interraciales e interculturales directas. Finalmente, los participantes valoraron el arte como una vía de acceso al conocimiento de los problemas de la realidad actual y como herramienta de transformación social. El arte tiene un gran potencial para conectar con la realidad, permite discutir, reflexionar y dialogar (Murillo, Riaño y Berbel, 2019).

De forma general, se puede afirmar que el profesorado en formación manifestó que la acción en la que participaron fue relevante, de acuerdo con los aspectos metodológicos relacionados con su futura práctica docente, en el modo de abordar prácticas creativas y musicales y en el potencial transformador del arte en este tipo de propuestas. Las estrategias utilizadas y los talleres realizados no solo son una oportunidad para vivir experiencias en primera persona, sino que pueden ayudarles a construir su mirada desde una perspectiva diferente a la hora de desarrollar propuestas artístico-musicales en su futuro profesional.

Tal como se ha mencionado, esta experiencia ha pretendido ofrecer oportunidades al alumnado universitario para que se formen en lo disciplinario y lo pedagógico de forma integrada, partiendo de un aprendizaje situado y cuyo planteamiento metodológico se basa en el aprendizaje basado en la creación ( $\mathrm{ABC}$ ) y el aprendizaje basado en proyectos (ABP). Como aportación del estudio, cabe señalar su abordaje desde un enfoque de aprendizaje que, además, sea Contextualizado, Artístico y Colaborativo (ABCAC). Su fin último es ofrecer estrategias que tengan en cuenta el desarrollo de la creatividad y que puedan ser utilizadas por los docentes, sean generalistas o educadores musicales, en una sociedad que cada día más requiere la conexión entre la escuela, la sociedad y la universidad.

\section{Financiación y agradecimientos}

Este proyecto ha sido financiado por FEDER/Ministerio de Ciencia, Innovación y Universidades-Agencia Estatal de Investigación/_EDU2017-84750-R) (FEDER/MCIU/AEI).

@ Noemy Berbel Gómez, Adolf Murillo Ribes y Ma Elena Riaño Galán. The content of this article is the sole responsibility of the authors. The Revista Electrónica de LEEME and Universitat de València are not liable for any legal actions that may arise involving the article's content. Revista Electrónica de LEEME - Lista Electrónica Europea de Música en la Educación-. http://ojs.uv.es/index/php/LEEME/index ISSN: 1575-9563. Editores: Universidad de Valencia y Jesús Tejada. Visibilidad de esta revista: SCOPUS, Emerging Sources Citation Index (Clarivate), EBSCO, CINDOC (CSIC), Citefactor, COPAC, Dialnet, DICE (CSIC), DOAJ, e-revistas (CSIC), EBSCO Premier, ERIH+, Gale Cengage Learning, IN-RECS, IRESIE, LATINDEX, MIAR, OCLC Worldcat, RESH, REDIB, RILM Core Journals, SUDOC, ULRICHS. Esta revista es de acceso libre mediante licencia Creative Commons 4.0 CC by. Política de archivado: etiqueta verde SHERPA-ROMEO. 


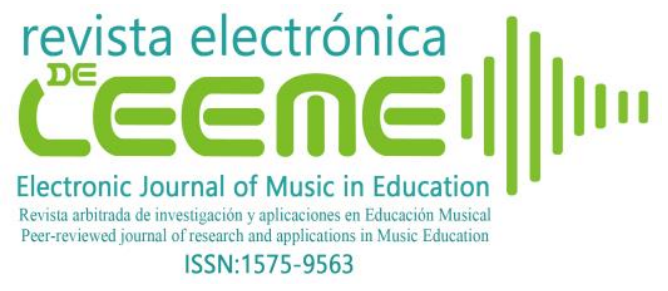

Noemy Berbel Gómez, Adolf Murillo Ribes y Mª Elena Riaño Galán Cuando el barrio educa: aprendizaje situado y creación artística colaborativa como herramienta en la formación musical (Revista Electrónica de LEEME) Número 46, pp. 68-91 https://ojs.uv.es/index.php/LEEME/index

ISSN:1575-9563

\section{Referencias}

Abrahams, F., Rowland, M. y Kohler, K. (2012). Music education behind bars: Giving voice to the inmates and the students who teach them. Music Educators Journal, 98(4), 67-73. doi: $10.1177 / 0027432112443711$

Anderson, W.M. y Campbell, P.S. (Eds.) (2011). Multicultural perspectives in music education (Vol.3). Plymouth, England: Rowman and Littlefield Education.

Amatea, E.S., Cholewa, B. y Mixon, K.A. (2012). Influencing preservice teachers' attitudes about working with low-income and/or ethnic minority families. Urban Education, 47, 801-834. doi:1177/0042085912436846

Aróstegui, J.L. y Cisneros-Cohernour, E. (2010). Reflexiones en torno a la formación del Profesorado de Música partir del análisis documental de los planes de estudio en Europa y América Latina. Profesorado. Revista de currículum y formación del profesorado, 14(2),179-189. Recuperado de: https://recyt.fecyt.es/index.php/profesorado/

Bartolome, S.J. (2017). Comparing field-teaching experiences: A longitudinal examination of preservice and first-year teacher perspectives. Journal of Research in Music Education, 65, 264-286. doi:10.1177/0022429417730043

Barrett, M.S., Zhukov, K. y Welch, G.F. (2019). Strengthening music provision in early childhood education: a collaborative self-development approach to music mentoring for generalist teachers. Music Education Research, 21(5), 529-548. doi:10.1080/14613808.2019.1647154

Biasutti, M. 2010. Investigating Trainee Music Teachers' Beliefs on Musical Abilities and Learning: A Quantitative Study. Music Education Research, 12(1), 47-69. doi:10.1080/14613800903568262

Biasutti, M., Hennessy, S. y de Vugt-Jansen, E. (2015). Confidence Development in Non-music Specialist Trainee Primary Teachers After an Intensive Programme. British Journal of Music Education, 32(2), 143-161. doi:10.1017/S0265051714000291

Bresler, L. (1993). Music in a double-bind: Instruction by non-specialists in elementary schools. Bulletin of the Council of Research in Music Education, 104, 1-13. doi:10.1080/10632913.1994.9936375

Brinkman, D.J. (2010). Teaching creatively and teaching for creativity. Arts Education Policy Review, 111, 48-50. doi:10.1007/978-3-319-40956_7

@ Noemy Berbel Gómez, Adolf Murillo Ribes y Mª Elena Riaño Galán. The content of this article is the sole responsibility of the authors. The Revista Electrónica de LEEME and Universitat de València are not liable for any legal actions that may arise involving the article's content. Revista Electrónica de LEEME - Lista Electrónica Europea de Música en la Educación-. http://ojs.uv.es/index/php/LEEME/index ISSN: 1575-9563. Editores: Universidad de Valencia y Jesús Tejada. Visibilidad de esta revista: SCOPUS, Emerging Sources Citation Index (Clarivate), EBSCO, CINDOC (CSIC), Citefactor, COPAC, Dialnet, DICE (CSIC), DOAJ, e-revistas (CSIC), EBSCO Premier, ERIH+, Gale Cengage Learning, IN-RECS, IRESIE, LATINDEX, MIAR, OCLC Worldcat, RESH, REDIB, RILM Core Journals, SUDOC, ULRICHS. Esta revista es de acceso libre mediante licencia Creative Commons 4.0 CC by. Política de archivado: etiqueta verde SHERPA-ROMEO. 
Burnard, P. (2012). Musical Creativities in Practice. Oxford: Oxford University Press.

Caeiro, R.M. (2018). Aprendizaje basado en la Creación y Educación Artística: proyectos de aula entre la metacognición y la metaemoción. Arte, Individuo y Sociedad, 30(1), 159177. doi:10.5209/ARIS.57043

Chou, H. (2007). Multicultural teacher education: Toward a culturally responsible pedagogy. Essays in Education, 21, 139-162. Recuperado de: https://openriver.winona.edu/cgi/viewcontent.cgi?article=1213\&context=eie

Christophersen, C. (2013). Perspectives on the Dynamics of Power within Collaborative Learning in Higher Music Education. En H. Gaund y H. Westerlund (Eds.), Collaborative Learning Higher Music Education (pp.77-87). London, UK: Asgate.

Craft, A. (2005). Creativity in Schools: Tensions and Dilemmas. Abingdon: Routledge.

Craft, A. (2012). Childhood in a digital age: creative challenges for educational futures. London Review of Education, 10(2), 173-190. doi: 10.1080/14748460.2012.691282

Creswell, J.W. (2013). Research design: qualitative, quantitative, and mixed methods approaches (Vol.4th). Thousand Oaks (California): SAGE.

Dey, I. (2005). Qualitative Data Analysis. A User-Friendly Guide for Social Scientists. London: Routledge.

de Vries, P. (2013) Generalist teachers' self-efficacy in primary school music teaching. Music Education Research, 15(4), 375-391. doi:10.1080/14613808.2013.829427

de Vries, P. (2014). Music without a Music Specialist: A Primary School Story. International Journal of Music Education, 33(2), 210-221. doi:10.1177/0255761413515818

Doberneck, D.M., Glass, C.R. y Schweitzer, J. (2010). From rhetoric to reality: A typology of publically engaged scholarship. Journal of Higher Education Outreach Engagement, 14, 5-35. doi:10.1080/1461380990010206

Elisondo, R., Donolo, D. y Rinaudo, M.C. (2013). The Unexpected and Education: Curriculums for Creativity. Creative Education, 4(12), 11-15. doi:10.4236/ce.2013.412A2002

Gibbs, G. (2012). Análisis de datos cualitativos en investigación cualitativa. Madrid: Morata.

Hammersley, M. y Atkinson, P. (2009). Etnografía. Métodos de investigación. Barcelona: Paidós Básica.

@ Noemy Berbel Gómez, Adolf Murillo Ribes y Mª Elena Riaño Galán. The content of this article is the sole responsibility of the authors. The Revista Electrónica de LEEME and Universitat de València are not liable for any legal actions that may arise involving the article's content. Revista Electrónica de LEEME - Lista Electrónica Europea de Música en la Educación-. http://ojs.uv.es/index/php/LEEME/index ISSN: 1575-9563. Editores: Universidad de Valencia y Jesús Tejada. Visibilidad de esta revista: SCOPUS, Emerging Sources Citation Index (Clarivate), EBSCO, CINDOC (CSIC), Citefactor, COPAC, Dialnet, DICE (CSIC), DOAJ, e-revistas (CSIC), EBSCO Premier, ERIH+, Gale Cengage Learning, IN-RECS, IRESIE, LATINDEX, MIAR, OCLC Worldcat, RESH, REDIB, RILM Core Journals, SUDOC, ULRICHS. Esta revista es de acceso libre mediante licencia Creative Commons 4.0 CC by. Política de archivado: etiqueta verde SHERPA-ROMEO. 


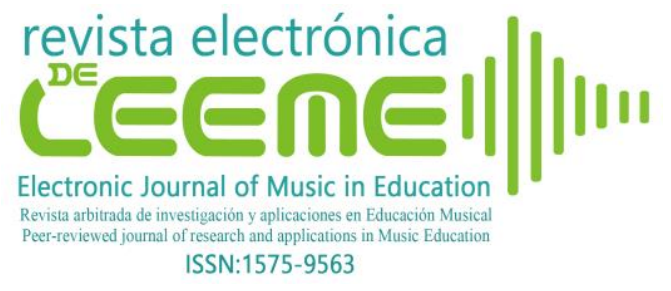

Noemy Berbel Gómez, Adolf Murillo Ribes y Mª Elena Riaño Galán

Cuando el barrio educa: aprendizaje situado y creación artística colaborativa como herramienta en la formación musical (Revista Electrónica de LEEME) Número 46, pp. 68-91 https://ojs.uv.es/index.php/LEEME/index

ISSN:1575-9563

Harris, A. y de Bruin, L.R. (2018). Training teachers for twenty-first century creative and critical thinking: Australian implications from an international study. Teaching Education, 29(3), 234-250. doi:10.1080/10476210.2017.1384802

Harris, A. (2014). The creative turn. Rotterdam: Sense Publishers.

Haston, W. y Russell, J.A. (2012). Turning into teachers: Influences of authentic context learning experiences on occupational identity development of preservice music teachers. Journal of Research in Music Education, 59, 369-392. doi: $10.1177 / 002242911414716$

Henley, J. (2015). Music: Naturally Inclusive, Potential Exclusive? En J.M. Deppeler, T. Loreman, R.A.L. Smith y L. Florian (Eds.), Inclusive Pedagogy Across the Curriculum, Vol. 6 (pp.161-186). Bingley: Emerald Publishing Group Limited.

Hennessy, S. (2017). Approaches to Increasing the Competence and Confidence of Student Teachers to Teach Music in Primary Schools. Education, 3-13, 45(6), 689-700. doi:10.1080/03004279.2017.1347130.

Hernández-Ponce, L.E., Rodríguez, J.C. y Giménez, C. (2011). La posibilidad del cambio social: aproximación psicosocial a los conceptos de agencia, empoderamiento y participación. Temas de coyuntura, 62, 79-102. Recuperado de: http://revistasenlinea.saber.ucab.edu.ve/temas/index.php/temasdecoyuntura/article/view 11183

Hickey, M. (2012). Music outside the lines: Ideas for composing in K-12 music classrooms. Oxford, UK: Oxford University Press.

Hutchins, E. (1995). Cognition in the Wild. Cambridge, MA: MIT Press.

Iyer, V. (2002). Embodied mind, situated cognition, and expressive microtiming in AfricanAmerican music. Music Perception, 19(3), 387-414. doi:10.1525/mp.2002.19.3.387

Kenny, A. (2015). Beginning a journey with music education: Voices from pre-service primary teachers. Music Education Research, 19(2), 111-122. doi:10.1080/14613808.2015.1077801

Kenny, A., Finneran, M. y Mitchell, E. (2015). Becoming an Educator in and Through the Arts: Forming and Informing Emerging Teachers' Professional Identity. Teaching and Teacher Education, 49, 159-167. doi:10.1016/j.tate.2015.03.004

@ Noemy Berbel Gómez, Adolf Murillo Ribes y Mª Elena Riaño Galán. The content of this article is the sole responsibility of the authors. The Revista Electrónica de LEEME and Universitat de València are not liable for any legal actions that may arise involving the article's content. Revista Electrónica de LEEME - Lista Electrónica Europea de Música en la Educación-. http://ojs.uv.es/index/php/LEEME/index ISSN: 1575-9563. Editores: Universidad de Valencia y Jesús Tejada. Visibilidad de esta revista: SCOPUS, Emerging Sources Citation Index (Clarivate), EBSCO, CINDOC (CSIC), Citefactor, COPAC, Dialnet, DICE (CSIC), DOAJ, e-revistas (CSIC), EBSCO Premier, ERIH+, Gale Cengage Learning, IN-RECS, IRESIE, LATINDEX, MIAR, OCLC Worldcat, RESH, REDIB, RILM Core Journals, SUDOC, ULRICHS. Esta revista es de acceso libre mediante licencia Creative Commons 4.0 CC by. Política de archivado: etiqueta verde SHERPA-ROMEO. 
Lave, J. y Wenger, E. (1991). Situated Learning. Legitimate Peripheral Participation. Cambridge: Cambridge University Press.

Leman, M. (2007). Embodied Music Cognition and Mediation Technology. Cambridge: MIT Press.

Mayorga, M.J. y Madrid, D. (2010). Modelos didácticos y Estrategias de enseñanza en el Espacio Europeo de Educación Superior. Tendencias Pedagógicas, 15(1), 91-111. Recuperado de: https://dialnet.unirioja.es/servlet/articulo?codigo=3221568

Marsh, K. y Young, S. (2006). Musical play. En G.E. McPherson (Ed.), The child as musician. A handbook of musical development (pp.298-310). New York: Oxford University Press.

Marsh, K. y Marsh, K.M. (2008). The musical playground: Global tradition and change in children's songs and games. New York: Oxford University Press.

McKoy, C. (2013). Effects of selected demographic variables on music student teachers' selfreported cross-cultural competence. Journal of Research in Music Education, 60, 375394. doi: $10.1177 / 0022429412463398$

Mota, G. (2015). Twenty-five Years of Music Teacher Education in Portugal - Revisiting History. En S. Figueiredo, J. Soares, y R.F. Schambeck (Eds.), The Preparation of Music Teachers: A Global Perspective (pp.241-264). Florianópolis: UDESC (Grupo de Pesquisa Música e Educação).

Murillo, A., Riaño, M. E. y Berbel, N. (2019). El aula como caja de resonancia para la creación sonora: nuevas arquitecturas y herramientas tecnológicas para acercar el arte sonoro al ámbito educativo. Revista Electrónica de LEEME, 43, 1-18 doi:10.7203/LEEME.43.14007

O'Neill, S.A. (2012). Personhood and music learning: Connecting perspectives and narratives, Research to practice. Waterloo, Ontario, Canada: Canadian Music Educators' Association.

O’Neill, S.A. (2014). Mind the gap: Transforming music engagement through learner-centred informal music learning. The Recorder: Journal of the Ontario Music Educators' Association, 56(2), 18-22. Recuperado de: https://www.researchgate.net/publication/273319581

@ Noemy Berbel Gómez, Adolf Murillo Ribes y Mª Elena Riaño Galán. The content of this article is the sole responsibility of the authors. The Revista Electrónica de LEEME and Universitat de València are not liable for any legal actions that may arise involving the article's content. Revista Electrónica de LEEME - Lista Electrónica Europea de Música en la Educación-. http://ojs.uv.es/index/php/LEEME/index ISSN: 1575-9563. Editores: Universidad de Valencia y Jesús Tejada. Visibilidad de esta revista: SCOPUS, Emerging Sources Citation Index (Clarivate), EBSCO, CINDOC (CSIC), Citefactor, COPAC, Dialnet, DICE (CSIC), DOAJ, e-revistas (CSIC), EBSCO Premier, ERIH+, Gale Cengage Learning, IN-RECS, IRESIE, LATINDEX, MIAR, OCLC Worldcat, RESH, REDIB, RILM Core Journals, SUDOC, ULRICHS. Esta revista es de acceso libre mediante licencia Creative Commons 4.0 CC by. Política de archivado: etiqueta verde SHERPA-ROMEO. 


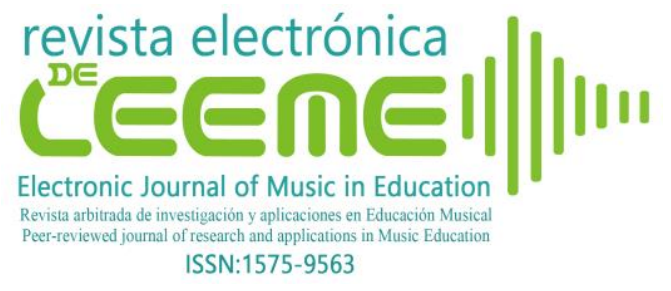

Noemy Berbel Gómez, Adolf Murillo Ribes y Mª Elena Riaño Galán Cuando el barrio educa: aprendizaje situado y creación artística colaborativa como herramienta en la formación musical (Revista Electrónica de LEEME) Número 46, pp. 68-91 https://ojs.uv.es/index.php/LEEME/index

ISSN:1575-9563

O’Neill, S.A. (2015). Youth Empowerment and Transformative Music Engagement. En C. Benedict, P. Schmidt, G. Spruce y P. Woodford (Ed.), The Oxford Handbook of Social Justice in Music Education (pp.388-405). New York: Oxford University Press.

O'Neill, S.A. (2016). Transformative music engagement and musical Flourishing. En G. McPherson (Ed.), The child as musician. A handbook of musical development (pp.606625). Oxford: Oxford University press.

Regelski, T.A. (2005). Music and music education: theory and practice to "make a difference". En D.K. Lines (Ed.), Music Education for the New Millenium (pp. 21-45). Oxford: Blackwell Publishing.

Reynolds, A.M., Jerome, A., Preston, A.L. y Haynes, H. (2005). Service-learning in music education: Participants' reflections. Bulletin of the Council for Research in Music Education, 165, 79-91. Recuperado de: https://www.jstor.org/stable/40319272

Robinson, N.R. (2016). Developing a critical consciousness for diversity and equity among preservice music teachers. Journal of Music Teacher Education, 26, 11-26. doi: $10.1177 / 1057083716643349$

Salvà-Tomàs, P.A., Jaume-Adrover, M. y Berbel-Gómez, N. (2019). Passat, present i desig de futur del Nou Llevant- Soledat Sud: projecte artístic transdisciplinar 'Re-habitar el Barrio'. Anuari d'Educació de les Illes Balears 2019, 2019, 252-266. Recuperado de: https://bit.ly/36LtcTC

Vergara, J.J. (2015). Aprendo porque quiero. El Aprendizaje Basado en Proyectos (ABP), paso a paso. Madrid: SM.

Wegerif, R. (2013). Dialogic: Education for the Internet Age. London: Routledge.

Wilson, G.B. y MacDonald, R.A.R. (2012). The sign of silence: negotiating musical identities in an improvising ensemble. Psychology of Music, 40(5), 1-16. doi:10.1177/0305735612449506

Young, G. (2018). Creative Interdisciplinary in the arts. En N.H. Hensel (Ed.), Exploring, Experiencing, and Envisioning Integration in US Arts Education (pp.15-26). Los Angeles: Palgrave.

@ Noemy Berbel Gómez, Adolf Murillo Ribes y Ma Elena Riaño Galán. The content of this article is the sole responsibility of the authors. The Revista Electrónica de LEEME and Universitat de València are not liable for any legal actions that may arise involving the article's content. Revista Electrónica de LEEME - Lista Electrónica Europea de Música en la Educación-. http://ojs.uv.es/index/php/LEEME/index ISSN: 1575-9563. Editores: Universidad de Valencia y Jesús Tejada. Visibilidad de esta revista: SCOPUS, Emerging Sources Citation Index (Clarivate), EBSCO, CINDOC (CSIC), Citefactor, COPAC, Dialnet, DICE (CSIC), DOAJ, e-revistas (CSIC), EBSCO Premier, ERIH+, Gale Cengage Learning, IN-RECS, IRESIE, LATINDEX, MIAR, OCLC Worldcat, RESH, REDIB, RILM Core Journals, SUDOC, ULRICHS. Esta revista es de acceso libre mediante licencia Creative Commons 4.0 CC by. Política de archivado: etiqueta verde SHERPA-ROMEO. 